\title{
LncRNA FAM83H-AS1 Amplification is Associated With a Poor Prognosis in Lung Adenocarcinoma and Can Serve as A Therapeutic Target
}

\section{Siwei Wang}

Nanjing Medical University Second Affiliated Hospital

Chencheng Han

Nanjing Medical University

Tongyan Liu

Jiangsu Cancer Hospital

Zhifei Ma

Nanjing Medical University

Mantang Qiu

Peking University

Jie Wang

Jiangsu Cancer Hospital

Xiufen Zheng

Jiangsu Cancer Hospital

Weizhang Xu

Jiangsu Cancer Hospital

Wenjia Xia

Jiangsu Cancer Hospital

Youtao Xu

Jiangsu Cancer Hospital

Jingwen $\mathrm{Hu}$

Jiangsu Cancer Hospital

Lin Xu

Jiangsu Cancer Hospital

Rong Yin ( $\square$ rong_yin@njmu.edu.cn )

Jiangsu cancer hospital

\section{Research}

Keywords: Long non-coding RNA, Machine learning, Lung adenocarcinoma, FAM83H-AS1 
Posted Date: July 23rd, 2020

DOI: https://doi.org/10.21203/rs.3.rs-45692/v1

License: (c) (i) This work is licensed under a Creative Commons Attribution 4.0 International License. Read Full License 


\section{Abstract}

Background: Few oncogenic drivers of long noncoding RNAs (IncRNAs) have been identified and investigated. Identifying noncoding drivers provides potential strategies for novel interventions in lung adenocarcinoma (LUAD).

Methods: We constructed a machine learning model for driver gene annotation using pan-cancer and clinical prognosis data from OncoKB and TCGA to predict potential oncogenic drivers of IncRNAs; then, we used zebrafish models to validate the biological function of candidate targets. The full length of FAM83H-AS1 was obtained by rapid amplification of the cDNA ends (RACE) assay. RNA pull-down, RNA immunoprecipitation (RIP), quantative mass spectrometry (QMS) and RNA sequencing (RNA-Seq) assays were utilized to explore the potential mechanisms. Additionally, we used CRISPR interference (CRISPRi) system and patient-derived tumor xenograft (PDTX) model to evaluate the therapeutic potential of targeting FAM83H-AS1 in vivo.

Results: The results suggested that FAM83H-AS1 was a potential oncogenic driver from the chromosome 8q24 amplicon; increases in the expression of FAM83H-AS1 resulted in poor prognosis for LUAD patients both in JSCH and TCGA cohorts. Functional assays revealed that FAM83H-AS1 promotes malignant progression and inhibits apoptosis. Mechanistically, FAM83H-AS1 binds with HNRNPK to enhance the translation of oncogenes RAB8B and RAB14. Experiments using CRISPR interference (CRISPRi)-mediated xenografts and patient-derived tumor xenograft (PDTX) models indicated that targeting FAM83H-AS1 inhibited LUAD progression in vivo.

Conclusions: Our work demonstrated that FAM83H-AS1 is a potential oncogenic driver that inhibits LUADmediated apoptosis via the FAM83H-AS1-HNRNPK-RAB8B/RAB14 axis. Importantly, we suggest targeting of FAM83H-AS1 as a potential therapeutic strategy for LUAD.

\section{Background}

Identifying cancer driver genes is essential for precision oncology. Somatic mutations in driver genes have been revealed across multiple types of cancers [1,2], and a number of these driver genetic alterations have become therapeutic targets or prognostic markers [3]. Recently, somatic copy number alterations (SCNAs) have been found to affect a larger fraction of cancer genomes than any other type of somatic genetic alterations, and these frequently altered genomic regions have critical roles in activating and inactivating oncogenic pathways [4]. In lung cancer, the TracerX program demonstrated that a high frequency of SCNAs, but not somatic mutations, is significantly correlated with a poor survival rate [5]. Therefore, among the considerable number of genes located in SCNA regions, novel cancer drivers should be further investigated.

Long noncoding RNAs (IncRNAs) play critical roles in cancer development, and the expression levels of IncRNAs is closely associated with oncogenic functions. Several oncogenic IncRNAs, such as FAL1 and PRAL, have been found to be regulated by SCNAs $[6,7]$. According to expression profiles of matched 
clinical and SCNA data, oncogenic drivers of IncRNAs can be distinguished from passengers by mathematical methods [8]. However, few IncRNAs have been identified as oncogenic drivers in lung adenocarcinoma (LUAD), which is the leading cause of cancer-associated deaths worldwide and accounts for nearly $40 \%$ of all lung cancer cases [9]. Therefore, systematic exploration and identification of noncoding drivers of LUAD is warranted.

The expression levels of oncogenic drivers of IncRNA have been thought to be regulated by corresponding genomic alterations [8]. In addition, multidimensional data, including clinical prognosis data and gene expression and SCNA data, could increase the ability to detect potential oncogenic drivers. Machine learning is a great method for constructing classifiers to identify oncogenic drivers [10]. Based on genomic data, a machine learning method was demonstrated to have obvious advantages in estimating prognostic signatures in transcription and methylation data [11-13]. Using in vivo and in vitro functional assays, the performance of machine learning algorithms could be assessed, and identified oncogenic drivers could also be validated.

In the current study, we extracted SCNA data, gene expression data and clinical prognosis data from The Cancer Genome Atlas (TCGA) LUAD population. Using a decision tree machine learning method, several potential oncogenic drivers of IncRNAs were identified. We further characterized the IncRNA FAM83H-AS1, which is highly expressed in LUAD tumor tissues and is associated with frequent $8 q 24$ amplification and poor prognosis. The characteristics of FAM83H-AS1 were subsequently validated in independent cohorts and with additional public datasets. Experimental investigation revealed that FAM83H-AS1 could bind with heterogeneous nuclear ribonucleoprotein K (HNRNPK) and increase the protein levels of RAB8B and RAB14, thus suppressing apoptosis and promoting tumorigenesis of LUAD cells. Importantly, targeting FAM83H-AS1 significantly reduced LUAD growth in a patient-derived tumor xenograft (PDTX) model.

\section{Materials And Methods}

\section{Identification of differentially expressed IncRNAs}

RNA sequencing data from the TCGA LUAD dataset were downloaded from the data portal (https://portal.gdc.cancer.gov) for 585 LUAD patients, including 56 normal lung tissue samples. The R package DESeq2 [14] was applied to HTSeq count data, and it detected 7320 differentially expressed genes $(P<0.01$ and fold change $>2.0)$ among 60483 genes. According to the "Gene_type" annotation by the Ensembl genes database, 596 IncRNAs were screened from differentially expressed genes. Two independent datasets, GSE74095 and GSE12236, were obtained from Gene Expression Omnibus (GEO) (https://www.ncbi.nlm.nih.gov/gds) for use.

\section{Identification of somatic copy number alterations}

The SCNA profiles of 513 LUAD patients in TCGA were obtained from the data portal (http://gdac.broadinstitute.org/runs). The total SCNA profiles were based on Affymetrix SNP 6.0 with removal of germline variation (http://gdac.broadinstitute.org/runs/stddata_2016_01_28/data/LUAD/), 
and the focal level SCNA profiles were identified by the GISTIC algorithm [46] (http://gdac.broadinstitute.org/runs/analyses_2016_01_28/data/LUAD/). The independent GSE29065 and GSE28572 datasets and the corresponding prognosis data were obtained from GEO. Additionally, clinical characteristics of patients, including overall survival (OS) and disease-free survival (DFS), were obtained from cBioPortal (http://www.cbioportal.org).

\section{Driver gene annotation and machine learning classifier}

The oncogenic annotations of protein-coding genes (PCGs) were obtained from the OncoKB database [3]. Among 290 PCGs annotated with oncogenic genomic variation, we classified PCGs according to whether or not they exhibited amplification to construct the decision tree model. We used the J48 decision tree function in the Weka package [15] to construct a pruned decision tree; we used the feature matrix as input and oncogenic driver annotation as the class variable. Subsequently, LUAD-upregulated IncRNAs were classified by the decision tree to discover candidate oncogenic drivers. In detail, total SCNA and focal SCNA profiles were both included, and Pearson's correlation analyses were performed between SCNA and expression profiles.

\section{Tissue samples and microarrays}

All primary LUAD tissues and adjacent normal tissues were collected from patients who had undergone surgery at the Department of Thoracic Surgery, The Affiliated Cancer Hospital of Nanjing Medical University (Jiangsu Cancer Hospital, Nanjing, China). All included tissue samples were confirmed by experienced pathologists and conducted in accordance with the International Ethical Guidelines for Biomedical Research Involving Human Subjects. Written informed consent was obtained from all patients. This study was approved by the Ethics Committee of the Affiliated Cancer Hospital of Nanjing Medical University. Tissue microarray (TMA) was constructed as described previously [16]. 68 pairs of lung cancer tissues and adjacent normal tissues from Jiangsu Cancer Hospital (JSCH) cohort were used to construct the TMA. RNA chromogenic in situ hybridization (CISH) was performed to detect FAM83HAS1 expression in TMA using digoxigenin-labeled probe (C10910 Inc1100151, RiboBio). According to percentages of positive stained cancer cells and areas, the $\mathrm{CISH}$ score was rated on a scale of one to twelve as described previously [16]. The characteristic and prognostic information of patients included in this study was obtained from follow-up team of Jiangsu Cancer Hospital.

\section{Cell culture, cell proliferation, colony formation and apoptosis assays}

All cell lines [A549, H1299, H1650, SPC-A1, H1975, H358, PC9 and human bronchial epithelial cell (HBE)] were purchased from Shanghai Institutes for Biological Science (Shanghai, China). A549, H1650, SPC-A1, H1975, H358 and HBE were cultured in DMEM medium (KeyGene, Nanjing, China); H1299 and PC9 were cultured in RPMI1640 medium (KeyGene), supplemented with 10\% FBS with $100 \mu / \mathrm{ml}$ penicillin and $100 \mathrm{mg} / \mathrm{ml}$ streptomycin included. All cell lines were grown in humidified air at $37 \mathrm{C}^{\circ}$ with $5 \% \mathrm{CO}_{2}$. Cell cultures were occasionally tested for mycoplasma (last tested December 2018). Authentication of cells 
was verified by short tandem repeat DNA profiling within 6 months, and cells used in experiments were within 10 passages from thawing.

Cell proliferation was examined using a CCK-8 Kit (Roche Applied Science) and Real time xCELLigence analysis system (RTCA) following the research protocol afforded by the manufacturer (ACEA Biosciences). Colony formation assays were performed to monitor LUAD cell cloning capability. Flow cytometer (FACScan; BD Biosciences) equipped with CellQuest software (BD Biosciences) was used to detect apoptosis level.

\section{RNA extraction, genome DNA extraction,Western blot and qRT-PCR analysis, andnuclear and cytoplasmic fractions extraction}

RNA extraction, DNA extraction, and qRT-PCR were performed as described previously [16]. GAPDH, $\beta$ Actin and snRNA U6 were used as internal controls. All primer sequences were listed in Additional file 1: Table S1. Protein was extracted from transfected cells and quantified as previously described [17] using $12 \%$ or $4 \%-20 \%$ poly-acrylamide gradient SDS gel. All antibodies were listed in Additional file 1: Table S2. RNA and protein isolation of nuclear and cytoplasmic fractions were applied with using PARIS Kit according to the manufacturer's protocol (Ambion, Life Technologies).

\section{SiRNA and plasmid construction and cell transfection}

The siRNAs were provided by Realgene Biotechnology (Nanjing, China). The full-length cDNA of human FAM83H-AS1 was synthesized and cloned into the expression vector pCDNA3.1 by Vigene Bioscience (Jinan, China). The final construct was verified by sequencing. SiRNA and plasmid vectors transfection was performed as described previously [16]. All siRNA sequences used are listed in Additional file 1: Table S3.

\section{RACE (Rapid amplification of cDNA ends)}

5'-RACE, 3'-RACE, and full-length amplification of FAM83H-AS1 were performed using a SMART RACE cDNA Amplification Kit (Clontech) according to the manufacturer's instructions. The gene-specific primers used for RACE analysis are presented in Additional file 1: Table S1.

\section{RNA immunoprecipitation and pull-down assays}

RNA immunoprecipitation was performed as described previously [16], and magnetic beads were conjugated with anti-HNRNPK or control anti-IgG antibody. In vitro translation assays were performed using mMESSAGE mMACHINE T7 Transcription Kit (Invitrogen) according to the manufacturer's instructions. Then, FAM83H-AS1 RNAs were labeled with desthiobiotinylation using the Pierce RNA 3' End Desthiobiotinylation Kit (Magnetic RNA-Protein Pull-Down Kit, Components; Thermo Fisher). RNA pulldown assays were performed with Magnetic RNA-Protein Pull-Down Kit according to the manufacturer's instructions. After elution of IncRNA-interacting proteins, they were subjected to mass spectrometric 
analysis. Liquid chromatography mass spectrometry (LC-MS) experiments were performed with a linear ion trap quadrupole mass spectrometer (Thermo Finnigan) equipped with a micro-spray source.

\section{Luciferase reporter assays}

The mRNA internal ribosome entry segment (IRES) of RAB8B and RAB14 was predicted by IRESite (http://iresite.org). The HNRNPK-binding sites of RAB8B and RAB14 mRNA were identified by the Blast program. The sequences of different fragments were synthesized and then inserted into the pGL3-basic vector (Vigene Bioscience). All constructs were verified by sequencing, and luciferase activity was assessed using the Dual Luciferase Assay Kit (Promega) according to the manufacturer's instructions.

\section{RNA sequencing and quantitative mass spectrometry}

A549 cells were plated in a 6-well plate and transfected with an siRNA targeting FAM83H-AS1 or a negative control. Twenty-four hours after transfection, cells were harvested for RNA extraction and subsequent library construction and sequencing (CapitalBio Technology, Beijing, China). Similarly, cells were harvested for protein extraction and subsequent iTRAQ (Isobaric Tag for Relative Absolute Quantitation)/TMT (Tandem Mass Tags) detection (PTM Bio, Hangzhou, China).

\section{CRISPR interference (CRISPRi)-mediated generation of FAM83H-AS1 knockdown LUAD cells}

For the CRISPRi experiments, six paired small guide RNAs (sgRNAs) were designed to target near the transcription start site (TSS) of FAM83H-AS1 (within 250 bp upstream and downstream). The location of the TSS was determined using NCBI (http://www.ncbi.nlm.nih.gov/). The sgRNA oligos were designed, phosphorylated, annealed, and cloned into a pBHCas-ZXS 023 vector using a BsmBI ligation strategy. Additional details and a list of the sgRNA sequences can be found in Additional file 1: Table S1.

\section{In vivo tumor growth assays, tumor engraftment, and PDTX maintenance}

All animal experiments were approved by the Nanjing Medical Experimental Animal Care Commission. The zebrafish tumor model was constructed according to the previous study [18]. In brief, 4 I $10^{2}$ A549 cells of control or silenced group were labeled by CellTracker CM-Dil (Invitrogen), and zebrafish embryos were monitored 96 hours for investigating tumor invasion and metastasis using a fluorescent microscope. BALB/c nude mice (4 to 6 weeks), purchased from the Vital River Laboratory Animal Technology (Beijing, China), were maintained under specific pathogen-free conditions. For the tumor formation assay, $10^{6} \mathrm{CRISPRi}$ constructed or control cells were subcutaneously injected into one flank of each mouse. Tumor volume was calculated using the following equation: $V=0.5$ I $D I$ Í $d^{2}(V$, volume; $D$, longitudinal diameter; $d$, transverse diameter). The method of building PDTX model has been described in the previous study [16].

\section{Statistical analysis}


GraphPad prism 8, R software version 3.5.1 and SPSS 23 were used to plot the figures. Differences between groups were assessed by two-tailed Student's $t$ test. The strength of the association between continuous variables was tested with Pearson's correlation test. Uni- and Multi-variate Cox regressions were used to identify independent risk factors of LUAD. For survival analysis, overall survival was calculated using the Kaplan-Meier method and the log-rank test. All $P$ values were two sided and $P$ value $<0.05$ were considered to be statistically significant.

\section{Results}

\section{A machine learning classifier identified candidate oncogenic drivers of IncRNAs in LUAD}

OncoKB oncogenic driver annotation was used with TCGA LUAD data to build a type of machine learning classifier known as the dichotomous decision tree. In detail, OncoKB annotated PCGs that harbored genomic amplicons were considered to be amplified oncogenic drivers, and then SCNA, gene expression and prognosis data for the PCG drivers were organized according to TCGA datasets (Additional file 2: Fig. S1a). In the screening phase, upregulated IncRNAs in LUAD were narrowed by the decision tree classifier to discover candidate oncogenic drivers of IncRNAs. Five-dimensional data resources were utilized with SCNA, gene expression and clinical prognosis data to construct the resulting tree, and this model had an accuracy of $81 \%$ in predicting the original 141 PCG drivers (Additional file 2: Fig. S1b).

SCNA profiles in different prognosis statuses are presented in Fig. 1, and total and focal SCNAs are shown in the upper and bottom heatmaps, respectively. These results suggested that amplified PCG drivers had significantly higher levels of accumulated SCNAs than other regions, and they exhibited a trend correlating with poor prognosis (Fig. 1a.c). The decision tree identified 72 candidate drivers of IncRNA expression, and they also harbored high levels of SCNA accumulation and strong correlation with poor prognosis (Fig. 1b.e). The decision tree classifier excluded the remaining 524 upregulated IncRNAs, and 75 of them were randomly selected to present in Fig. 1d. These results indicated that amplified oncogenic drivers of IncRNA had a higher level of SCNA accumulation (Additional file 2: Fig. S1c), an association with worse prognoses (Additional file 2: Fig. S1d) and a stronger correlation between SCNA and expression levels (Additional file 2: Fig. S1e). Among these candidate oncogenic drivers of IncRNAs, 18 IncRNAs were found to be located in high frequency amplified regions, and most of them (11 of 18) were grouped on chromosome 8q24 (Fig. 2a.b). We subsequently used zebrafish tumor models to quickly validate the biological functions of the four top ranked IncRNAs in vivo (Fig. 2C), and FAM83H-AS1 was shown to exhibit the most significant effect in promoting proliferation and metastasis.

\section{Chromosome 8q24 amplified FAM83H-AS1, leading to poor prognosis of LUAD}

We gained insights into the oncogenic functions of FAM83H-AS1 in LUAD cohorts and datasets. The expression of FAM83H-AS1 was analyzed in 40 pairs of primary LUAD and adjacent nontumor tissues from the Affiliated Cancer Hospital of Nanjing Medical University between August 1 and October 1 in 2017. FAM83H-AS1 was highly upregulated in LUAD, with an average fold change of $13.30(P<0.001)$ 
(Fig. 3a). TCGA and other datasets of gene expression, including, GSE74095 and GSE12236, all indicated that FAM83H-AS1 was overexpressed in LUAD tissues (Fig. 3b.c.d).

FAM83H-AS1 expression was then detected in the JSCH cohort by CISH using a TMA of 68 pairs of LUAD and adjacent nontumor tissues. Overexpression of FAM83H-AS1 in LUAD was validated by CISH scores from the TMA (Fig. 3e.f). Kaplan-Meier survival analysis showed that patients with a higher CISH score for FAM83H-AS1 had a shorter overall survival (Fig. 3h), and the result was validated by data from TCGA (Fig. 3j). The multivariable Cox proportional hazards model indicated that the FAM83H-AS1 level was an independent prognostic factor for LUAD patients (Fig. 3g; Additional file 1: Table S4). Additionally, the expression level of FAM83H-AS1 was positively correlated with tumor size and TNM stage in both the JSCH and TCGA cohorts (Fig. 3i;Additional file 3: Fig S2a.b). An increase in the SCNV for FAM83H-AS1 was shown to be related to poor prognosis in both the GSE29065 and GSE28572 datasets (Fig. 3k; Additional file 3: Fig. S2C). PCR results validated that the expression level of FAM83H-AS1 was positively correlated with the amplification level of FAM83H-AS1 (Fig. 3l), which was consistent with the TCGA data (Additional file 3: Fig. S2d.e). In addition, Cancer Cell Line Encyclopedia (CCLE) datasets indicated a strong positive relationship between the SCNV of FAM83H-AS1 and LUAD cell lines (Additional file 3: Fig. S2f).

\section{The high expression level of FAM83H-AS1 induced malignant behavior in LUAD cells}

The expression of FAM83H-AS1 was first detected in several LUAD cell lines and was found to be remarkably higher than it was in the normal bronchial epithelial cell line HBE (Fig. 4a). FAM83H-AS1 is located on chromosome 8q24 in humans; a transcript length of $2162 \mathrm{nt}$ was determined by 5 ' and $3^{\prime}$ RACE assays, which is slightly shorter than the full transcript length of $2743 \mathrm{nt}$ (NR_033849.1) (Fig. 4b; Additional file 2: Fig. S2g). Further, no translation of FAM83H-AS1 was found according to coding potentiality assay (Fig. 4 c).

To investigate the biological function of FAM83H-AS1, siRNA-mediated knockdown and plasmidmediated overexpression of FAM83H-AS1 were performed in LUAD cell lines (A549 and SPC-A1) to explore its pathophysiological significance (Fig. 4d; Additional file 4: Fig. S3a). Colony formation assays showed that the silencing and overexpression of FAM83H-AS1 suppressed and induced the clonogenicity of LUAD cells, respectively (Fig 4e; Additional file 4: Fig S3b). Using a flow cytometry assay, we determined that knockdown of FAM83H-AS1 greatly promoted apoptosis, whereas ectopic expression of FAM83H-AS1 impaired the apoptotic ability of A549 and SPC-A1 cells (Fig 4f; Additional file 4: Fig. S3c). Consistently, oncogenic functions of FAM83H-AS1 were indicated by PARP and Caspase-3 cleavage in apoptosis assays (Fig. $\mathbf{4 g}$ ). In addition, CCK-8 assay results indicated that the knockdown and upregulation of FAM83H-AS1 could obviously suppress and induce the proliferation of LUAD cells, respectively (Fig. 4h; Additional file 4: Fig. S3d). Transwell and Matrigel assays showed that FAM83H-AS1 markedly affected the migration and invasion abilities of A549 and SPC-A1 cell lines (Additional file 4: Fig. S3e). 
Although the head-to-head coding gene FAM83H has been known to be involved in the progression of human cancers $[19,20]$, we did not find any significant changes in FAM83H mRNA or protein in FAM83HAS1-reduced LUAD cells (Fig. 4i). Additionally, the oncogene MYC, which is located in 8q24 and is known to be a driver in human cancers $[4,21]$, revealed no significant changes after the silencing of FAM83HAS1 (Fig. 4i).

To explore the molecular mechanisms of FAM83H-AS1 promoting LUAD tumorigenesis, we first performed nuclear mass separation and FISH assays and found that FAM83H-AS1 was mainly distributed in the cell cytoplasm (Fig. 4j.k), which indicated that FAM83H-AS1 might exert biological function at the posttranscriptional level. A subsequent RNA pull-down experiment was performed to identify potential proteins binding with FAM83H-AS1 (Fig. 5a). Mass spectrometry analysis of a differentially displayed band revealed that HNRNPK was associated with FAM83H-AS1 (Fig. 5b). Then, we confirmed the association of FAM83H-AS1 and HNRNPK by western blot of the proteins isolated from the RNA pull-down assays (Fig. 5c). Additionally, a RIP assay was performed with an HNRNPK antibody to ensure that FAM83H-AS1 formed a complex with HNRNPK (Fig. 5d).

HNRNPK is an RNA-binding protein that is localized both in the cytoplasm and nucleus [22], and it has been shown to regulate the translation of oncogenes in cancer cells [23,24]. Western blot assays showed that FAM83H-AS1 did not affect the overall expression of HNRNPK within the A549 cells (Fig. 5e); however, FAM83H-AS1 overexpression increased HNRNPK expression within the cytoplasm, whereas FAM83H-AS1 knockdown had an opposite effect (Fig. 5e). Immunofluorescence assays validated these results (Fig. 5f). A further finding confirmed by RNA pull-down using biotinylated truncations of FAM83HAS1 was that the stem-loop structure from $1301 \mathrm{nt}$ to $1881 \mathrm{nt}$ ( $3^{\text {rd }}$ truncation) was sufficient for enabling interaction between FAM83H-AS1 and HNRNPK (Fig. 5g). In addition, western blotting demonstrated that this identified truncation of FAM83H-AS1 could suppress apoptosis at a rate similar to that of the full length FAM83H-AS1 (Fig. 5h).

\section{FAM83H-AS1 targeted RAB8B and RAB14 and promoted their translation}

To identify the potential downstream molecular targets of FAM83H-AS1, we conducted RNA-Seq and QMS assays after silencing FAM83H-AS1 in A549 cells. A total of 230 differentially expressed mRNAs (FDR $<0.01$ and fold change > 2) were detected (Fig. 6a; Additional file 1: Table S5; Additional file 4: Fig. S4a), and the QMS assay demonstrated 258 differentially expressed proteins (FDR $<0.05$ and fold change > 1.2; Fig. 6a; Additional file 1: Table S6; Additional file 4: Fig. S4b). However, few genes overlapped at both the mRNA and protein levels (Fig. 6B). Considering that the FAM83H-AS1 and HNRNPK complex is mainly distributed in the cytoplasm, we speculated that FAM83H-AS1 may affect the translation of downstream genes in LUAD cells.

HNRNPK has been shown to have a preference for AU/CU-rich sequences in 5' untranslated regions (UTRs) and to have a specified motif of $\mathrm{N}_{2-5} \mathrm{C}(\mathrm{C} / \mathrm{U}) \mathrm{ACC}(\mathrm{C} / \mathrm{A}) \mathrm{N}_{11-17}$ [25]. Therefore, the differentially expressed genes harboring the HNRNPK motif in their 5'UTRs were identified from both the RNA-Seq and 
QMS data, which indicated that HNRNPK-targeting genes were significantly enriched in the QMS results (Fig. 6c). Among the top ranked genes in the QMS results, no significant changes in expression level were revealed by RNA-Seq (Fig. 6d). Two known oncogenes, RAB8B and RAB14, of the RAS family of oncogenic proteins, were found to be significantly downregulated at the protein level after FAM83H-AS1 silencing. Considering the extremely low RNA levels of RAB8B and RAB14 and the effect of HNRNPK in stimulating the activity of mRNA IRESs to regulate translation $[26,27]$, we analyzed the 5'UTRs of RAB8B and RAB14, which indicated potential IRES segments harboring HNRNPK motifs (Fig. 6e). The RIP assays performed with HNRNPK antibody indicated that HNRNPK could bind to RAB8B and RAB14 mRNAs (Fig. 6f). Furthermore, we cloned the wild-type and mutated 5'UTRs of the RAB8B and RAB14 mRNAs and performed dual luciferase reporter assays with them. Compared with the control group, the overexpression of HNRNPK efficiently promoted luciferase activity of wild-type groups but not mutated groups (Fig. $\mathbf{6 g}$ ). These results suggested that the 5'UTRs of both RAB8B and RAB14 could be bound by HNRNPK. We also observed that the translation inhibitor cycloheximide inhibited the HNRNPK overexpression-induced increase in the protein levels of RAB8B and RAB14 in FAM83H-AS1-knockdown LUAD cells (Fig. 6h).

The silencing of FAM83H-AS1 decreased the expression of RAB8B and RAB14, whereas overexpressing FAM83H-AS1 increased the expression of these genes at the protein level (Fig. 6i; Additional file 5: Fig.

S4c). The high expression level of RAB14 has been reported to inhibit apoptosis in non-small cell lung cancer (NSCLC) [28], and we found that the silencing of RAB8B or RAB14 suppressed the clonogenicity of A549 cells (Fig. 6j). To determine whether FAM83H-AS1 inhibits LUAD cell apoptosis via the FAM83HAS1-HNRNPK-RAB8B/RAB14 axis, colony formation, flow cytometry assays and western blotting of cleaved PARP and Caspase-3 were performed; silencing of HNRNPK partially rescued the apoptosisinhibiting effect induced by FAM83H-AS1 (Fig. 6k.I.m). Additionally, the silencing of HNRNPK partially reversed the effects of FAM83H-AS1 on RAB8B and RAB14 (Fig. 6m).

\section{FAM83H-AS1 promoted LUAD in vitro and was revealed as a potential therapeutic target}

To validate the biological function of FAM83H-AS1 in vivo, we constructed A549 cells with CRISPRimediated FAM83H-AS1 silencing. A total of six sgRNAs around the transcription start site (TSS) of FAM83H-AS1 were designed to suppress the transcription of FAM83H-AS1 (Additional file 5: Fig. S4d) , and the combination of three sgRNAs in the 3'-end of TSS produced the highest knockdown efficiency (Additional file 5: Figure S4e) without affecting the expression of FAM83H (Additional file 5: Figure S4f). Consequently, xenograft tumor models demonstrated that the tumors derived from CRISPRi-mediated FAM83H-AS1 silenced A549 cells had a smaller tumor size than that of the control (Fig. 7a).

We then developed a PDTX model from four LUAD patients and evaluated the therapeutic potential of targeting FAM83H-AS1 by intratumor injection of cholesterol-conjugated siFAM83H-AS1 and a control siRNA (4 times and twice a week) (Fig. 7b.c). Immunohistochemistry (IHC) revealed that the siFAM83HAS1 group had fewer RAB8B- and RAB14-positive cells but more TdT-mediated dUTP nick end labeling (TUNEL)-positive cells than the control group (Additional file 5: Fig S4g). As a result, suppressing 
FAM83H-AS1 inhibited PDTX growth in vivo, suggesting that FAM83H-AS1 could serve as a promising therapeutic target for LUAD.

\section{Discussion}

In this study, we identified potential oncogenic drivers of IncRNAs in LUAD using machine learning algorithms. The oncogenic functions of four candidate IncRNAs located at 8q24 were tested in vivo using zebrafish models, and the molecular function of FAM83H-AS1 was revealed. In LUAD cells, FAM83H-AS1 bound with cytoplasmic HNRNPK to form an RNA-protein complex, which further bound to the 5'UTRs of target mRNAs RAB8B and RAB14. This interaction enhanced the translation of these oncogenes and upregulated their protein levels, which finally promoted the malignant progression of LUAD (Fig. 7d).

To identify oncogenic drivers of IncRNAs in cancers, genomic variation-associated data have been widely used in the discovery phase $[6-8,29]$. Integrative analysis of genomic and transcriptional data provided a theoretical basis for identifying these candidate drivers. Unlike PCGs, noncoding RNAs have been demonstrated to lack hotspot point mutations, but structural variants, including SCNAs, breakpoints and fusion events, have been thought to be substantial contributors to noncoding drivers [30]. High frequent SCNA gain or loss in the genome of cancers has now been revealed by TCGA project, with joint analyses performed on IncRNA profiles in several cancer types, including glioblastoma multiforme, ovarian cancer, lung squamous cell carcinoma (LSCC) and prostate cancer [8]. In addition, IncRNAs of RBPMS-AS1, TDRKH-AS1, LINC00578, RP11-470 M17.2 and LINC00941 were revealed to be key prognostic biomarkers of LUAD as a result of weighted gene coexpression and GISTIC analyses, but none of these IncRNAs has been validated in functional assays [29]. Chromosome 8q24 is a region with frequent SCNAs, regardless of arm or focal levels of LUAD [31], and it was also found to harbor most candidate drivers of IncRNAs in our study. This so-called "8q24 gene desert" was shown to be a hotspot region linking oncogenic IncRNAs and genomic variations [32-34], and this study added further insights into the oncogenic function of the $8 q 24$ amplicon in LUAD.

FAM83H-AS1 has been proven to have potent tumor-promoting activity in colorectal carcinoma, breast cancer, bladder cancer and NSCLC [35-38]. Zhang et al. found that the proliferation, migration and invasion of NSCLC cells were decreased after FAM83H-AS1 downregulation, which is consistent with our results [37]. However, the molecular function of FAM83H-AS1 in human cancer cells has not been uncovered, especially in LUAD. Furthermore, the upregulation of FAM83H-AS1 was demonstrated to be related to poor prognosis in lung cancer, ovarian cancer and gastric cancer patients [39, 40]. All these results indicated that FAM83H-AS1 has conserved oncogenic function among different types of malignant tumors, even though expression level varies greatly; however, mechanism investigation and functional experiments in vivo were rare to be conducted in these previous studies. In summary, the oncogenic IncRNA FAM83H-AS1 exhibits a trend of overexpression in human cancers; therefore, we considered that transcriptional activation could also contribute to the high expression level of FAM83HAS1. 
HNRNPK is a multifunctional protein that plays important roles in cancer cells. Previous studies found that HNRNPK could regulate biological processes at both transcriptional and posttranscriptional levels. For example, HNRNPK was shown to interact with the RNA polymerase II transcription machinery to stimulate transcription $[41,42]$ and to be involved in regulating the translation of MYC, P21 and ERK in cancer cells $[23,24,43]$. Additionally, HNRNPK was found to be essential for the anti-apoptosis mechanism in cancer cells, and it is independent of p53 status $[44,45]$. Furthermore, HNRNPK protein has been revealed to play a regulatory role in the molecular mechanisms of IncRNAs [46]. Previous studies discovered that HNRNPK is required for Xist-mediated chromatin modifications [47] and that it binds with IncRNA CASC11 and linc00460 to form RNA-protein complexes in colorectal and lung cancers, respectively $[48,49]$. In the current study, we identified a complex of FAM83H-AS1 and HNRNPK in LUAD cells. To control for possible confounding bias, we therefore used high-throughput methods at both the RNA and protein levels to elucidate the underlying molecular mechanisms.

In conclusion, we have identified FAM83H-AS1 as a potential oncogenic driver and described its regulatory function in malignant phenotypes, especially apoptosis and clonogenicity. Importantly, our study discovered that FAM83H-AS1 interacts with HNRNPK to promote the translation of RAB8B and RAB14 and that LUAD PDTX growth was inhibited by targeting FAM83H-AS1.

\section{List Of Abbreviations}

Long non-coding RNA, IncRNA; lung adenocarcinoma, LUAD; non-small cell lung cancer, NSCLC; rapid amplification of the cDNA ends, RACE; RNA immunoprecipitation, RIP; quantative mass spectrometry, QMS; patient-derived tumor xenograft, PDTX; somatic copy number alteration, SCNA; The Cancer Genome Atlas, TCGA; Gene Expression Omnibus, GEO; protein-coding genes, PCGs; tissue microarray; TMA; Jiangsu Cancer Hospital, JSCH; in situ hybridization; CISH; human bronchial epithelial cell, HBE; internal ribosome entry segment; IRES; liquid chromatography mass spectrometry, LC-MS; small guide RNAs, sgRNA; transcription start site, TSS; TdT-mediated dUTP nick end labeling, TUNEL; untranslated regions, UTR.

\section{Declarations}

\section{Acknowledgements}

Not applicable

\section{Authors' contributions}

WSW and YR conceived and designed the study. HCC, LTY, MZF, QMT, WJ, ZXF, XWZ, XWJ, XYT and HJW contributed to carry out the experiments. WSW and HCC contributed to data analysis. LTY and XL provided clinical samples and clinical information. WSW wrote the manuscript. YR and XL supervised the research. All authors read and approved the final manuscript. 


\section{Funding}

This work was supported by Key Project of Cutting-edge Clinical Technology of Jiangsu Province (BE2017759), the National Science Foundation of China (81672295, 81572261, 81802277, 81872378, 81802907, 81702265 and 81602014), China Postdoctoral Science Foundation (2018M642198 and 2018M640465), Jiangsu Province Postgraduate Innovation Programme (KYCX19_1174), and Project of Jiangsu Provincial Medical Talent (ZDRCA2016033).

\section{Availability of data and materials}

All data that support the findings of this study are available from the corresponding authors upon reasonable request.

\section{Ethics approval and consent to participate}

The research was approved by the Ethics Committee of the Affiliated Cancer Hospital of Nanjing Medical University.

\section{Consent for publication}

All subjects have written informed consent.

\section{Competing interests}

The authors declare that they have no competing interests.

\section{Author details}

${ }^{1}$ Department of Thoracic Surgery, Jiangsu Key Laboratory of Molecular and Translational Cancer Research, Nanjing Medical University Affiliated Cancer Hospital \& Jiangsu Cancer Hospital \& Jiangsu Institute of Cancer Research, Nanjing, China. ${ }^{2}$ Department of Science and technology, The Affiliated Cancer Hospital of Nanjing Medical University \& Jiangsu Cancer Hospital \& Jiangsu Institute of Cancer Research, Jiangsu Key Laboratory of Molecular and Translational Cancer Research, Nanjing, China. ${ }^{3}$ Department of Thoracic Surgery, Peking University People's Hospital, Beijing, China. ${ }^{4}$ Biobank of Lung Cancer, Jiangsu Biobank of Clinical Resources, Nanjing, China. ${ }^{5}$ Collaborative Innovation Center For Cancer Personalized Medicine, Nanjing Medical University, Nanjing China.

\section{References}

1. 1. Forbes SA, Bindal N, Bamford S, Cole C, Kok CY, Beare D, Jia M, Shepherd R, Leung K, Menzies A et al: COSMIC: mining complete cancer genomes in the Catalogue of Somatic Mutations in Cancer. Nucleic Acids Research 2010, 39(Database):D945-D950. 
2. 2. Pleasance ED, Cheetham RK, Stephens PJ, McBride DJ, Humphray SJ, Greenman CD, Varela I, Lin $\mathrm{ML}$, Ordonez GR, Bignell GR et al: A comprehensive catalogue of somatic mutations from a human cancer genome. Nature 2010, 463(7278):191-196.

3. 3. Chakravarty D, Gao J, Phillips SM, Kundra R, Zhang H, Wang J, Rudolph JE, Yaeger R, Soumerai T, Nissan MH et al: OncoKB: A Precision Oncology Knowledge Base. JCO Precis Oncol 2017, 2017.

4. 4. Zack TI, Schumacher SE, Carter SL, Cherniack AD, Saksena G, Tabak B, Lawrence MS, Zhsng CZ, Wala J, Mermel CH et al: Pan-cancer patterns of somatic copy number alteration. Nat Genet 2013, 45(10):1134-1140.

5. 5. Jamal-Hanjani M, Wilson GA, McGranahan N, Birkbak NJ, Watkins TBK, Veeriah S, Shafi S, Johnson DH, Mitter R, Rosenthal R et al: Tracking the Evolution of Non-Small-Cell Lung Cancer. $N$ Engl J Med 2017, 376(22):2109-2121.

6. 6. Hu X, Feng Y, Zhang D, Zhao SD, Hu Z, Greshock J, Zhang Y, Yang L, Zhong X, Wang L-P: A functional genomic approach identifies FAL1 as an oncogenic long noncoding RNA that associates with BMl1 and represses p21 expression in cancer. Cancer cell 2014, 26(3):344-357.

7. 7. Zhou CC, Yang F, Yuan SX, Ma JZ, Liu F, Yuan JH, Bi FR, Lin KY, Yin JH, Cao GW et al: Systemic genome screening identifies the outcome associated focal loss of long noncoding RNA PRAL in hepatocellular carcinoma. Hepatology 2016, 63(3):850-863.

8. 8. Du Z, Fei T, Verhaak RG, Su Z, Zhang Y, Brown M, Chen Y, Liu XS: Integrative genomic analyses reveal clinically relevant long noncoding RNAs in human cancer. Nat Struct Mol Biol 2013, 20(7):908-913.

9. 9. Siegel RL, Miller KD, Jemal A: Cancer statistics, 2018. CA: A Cancer Journal for Clinicians 2018, 68(1):7-30.

10. 10. Bailey MH, Tokheim C, Porta-Pardo E, Sengupta S, Bertrand D, Weerasinghe A, Colaprico A, Wendl MC, Kim J, Reardon B: Comprehensive characterization of cancer driver genes and mutations. Cell 2018, 173(2):371-385.

11. 11. Tang X-R, Li Y-Q, Liang S-B, Jiang W, Liu F, Ge W-X, Tang L-L, Mao Y-P, He Q-M, Yang X-J: Development and validation of a gene expression-based signature to predict distant metastasis in locoregionally advanced nasopharyngeal carcinoma: a retrospective, multicentre, cohort study. The Lancet Oncology 2018, 19(3):382-393.

12. 12. Zhou J, Yu L, Gao X, Hu J, Wang J, Dai Z, Wang J-F, Zhang Z, Lu S, Huang X: Plasma microRNA panel to diagnose hepatitis B virus-related hepatocellular carcinoma. J Clin Oncol 2011, 29(36):4781-4788.

13. 13. Qiu J, Peng B, Tang Y, Qian Y, Guo P, Li M, Luo J, Chen B, Tang H, Lu C: CpG methylation signature predicts recurrence in early-stage hepatocellular carcinoma: results from a multicenter study. $J$ Clin Oncol 2017, 35(7):734-742.

14. 14. Love MI, Huber W, Anders S: Moderated estimation of fold change and dispersion for RNA-seq data with DESeq2. Genome biology 2014, 15(12):550. 
15. 15. Frank E, Mark A, H. I: Data Mining: Practical Machine Learning Tools and Techniques 2016, 78(71):74-75.

16. 16. Qiu M, Xia W, Chen R, Wang $S$, Xu Y, Ma Z, Xu W, Zhang E, Wang J, Fang T: The circular RNA circPRKCl promotes tumor growth in lung adenocarcinoma. Cancer research 2018, 78(11):28392851.

17. 17. Chen R, Xia W, Wang S, Xu Y, Ma Z, Xu W, Zhang E, Wang J, Fang T, Zhang Qa: Long Noncoding RNA SBF2-AS1 Is Critical for Tumorigenesis of Early-Stage Lung Adenocarcinoma. Molecular Therapy-Nucleic Acids 2019, 16:543-553.

18. 18. Liu C, Zhang Y, Lim S, Hosaka K, Yang Y, Pavlova T, Alkasalias T, Hartman J, Jensen L, Xing X et al: A Zebrafish Model Discovers a Novel Mechanism of Stromal Fibroblast-Mediated Cancer Metastasis. Clin Cancer Res 2017, 23(16):4769-4779.

19. 19. Kim KM, Park SH, Bae JS, Noh SJ, Tao GZ, Kim JR, Kwon KS, Park HS, Park BH, Lee H et al: FAM83H is involved in the progression of hepatocellular carcinoma and is regulated by MYC. Scientific Reports 2017, 7(1):3274.

20. 20. Snijders AM, Lee SY, Hang B, Hao W, Bissell MJ, Mao JH: FAM83 family oncogenes are broadly involved in human cancers: an integrative multi-omics approach. Mol Oncol 2017, 11(2):167-179.

21. 21. Beroukhim R, Mermel CH, Porter D, Wei G, Raychaudhuri S, Donovan J, Barretina J, Boehm JS, Dobson J, Urashima $\mathrm{M}$ et al: The landscape of somatic copy-number alteration across human cancers. Nature 2010, 463(7283):899-905.

22. 22. Thompson PJ, Dulberg V, Moon KM, Foster LJ, Chen C, Karimi MM, Lorincz MC: hnRNP K coordinates transcriptional silencing by SETDB1 in embryonic stem cells. PLoS Genet 2015, 11(1):e1004933.

23. 23. Notari M, Neviani P, Santhanam R, Blaser BW, Chang J-S, Galietta A, Willis AE, Roy DC, Caligiuri MA, Marcucci G: A MAPK/HNRPK pathway controls BCR/ABL oncogenic potential by regulating MYC mRNA translation. Blood 2006, 107(6):2507-2516.

24. 24. Habelhah H, Shah K, Huang L, Ostareck-Lederer A, Burlingame A, Shokat KM, Hentze MW, Ronai Ze: ERK phosphorylation drives cytoplasmic accumulation of hnRNP-K and inhibition of mRNA translation. Nature cell biology 2001, 3(3):325-330.

25. 25. Yano $\mathrm{M}, \mathrm{Okano} \mathrm{HJ}$, Okano $\mathrm{H}$ : Involvement of $\mathrm{Hu}$ and heterogeneous nuclear ribonucleoprotein $\mathrm{K}$ in neuronal differentiation through p21 mRNA post-transcriptional regulation. The Journal of biological chemistry 2005, 280(13):12690-12699.

26. 26. Notari M, Neviani P, Santhanam R, Blaser BW, Chang JS, Galietta A, Willis AE, Roy DC, Caligiuri MA, Marcucci $G$ et al: A MAPK/HNRPK pathway controls BCR/ABL oncogenic potential by regulating MYC mRNA translation. Blood 2006, 107(6):2507-2516.

27. 27. Evans JR, Mitchell SA, Spriggs KA, Ostrowski J, Bomsztyk K, Ostarek D, Willis AE: Members of the poly $(\mathrm{rC})$ binding protein family stimulate the activity of the c-myc internal ribosome entry segment in vitro and in vivo. Oncogene 2003, 22(39):8012-8020. 
28. 28. Wang R, Wang ZX, Yang JS, Pan X, De W, Chen LB: MicroRNA-451 functions as a tumor suppressor in human non-small cell lung cancer by targeting ras-related protein 14 (RAB14). Oncogene 2011, 30(23):2644-2658.

29. 29. Wang L, Zhao H, Xu Y, Li J, Deng C, Deng Y, Bai J, Li X, Xiao Y, Zhang Y: Systematic identification of lincRNA-based prognostic biomarkers by integrating lincRNA expression and copy number variation in lung adenocarcinoma. Int J Cancer 2019, 144(7):1723-1734.

30. 30. Rheinbay E, Nielsen MM, Abascal F, Wala JA, Shapira O, Tiao G, Hornshoj H, Hess JM, Juul RI, Lin $Z$ et al: Analyses of non-coding somatic drivers in 2,658 cancer whole genomes. Nature 2020, 578(7793):102-111.

31. 31. Chen J, Yang H, Teo ASM, Amer LB, Sherbaf FG, Tan CQ, Alvarez JJS, Lu B, Lim JQ, Takano A et al: Genomic landscape of lung adenocarcinoma in East Asians. Nat Genet 2020.

32. 32. Huppi K, Pitt J, Wahlberg B, Caplen N: The 8q24 Gene Desert: An Oasis of Non-Coding Transcriptional Activity. Frontiers in Genetics 2012, 3(69).

33. 33. Xiang J-F, Yin Q-F, Chen T, Zhang Y, Zhang X-O, Wu Z, Zhang S, Wang H-B, Ge J, Lu X et al: Human colorectal cancer-specific CCAT1-L IncRNA regulates long-range chromatin interactions at the MYC locus. Cell Research 2014, 24(5):513-531.

34. 34. Ling H, Spizzo R, Atlasi Y, Nicoloso M, Shimizu M, Redis RS, Nishida N, Gafà R, Song J, Guo Z: CCAT2, a novel noncoding RNA mapping to 8q24, underlies metastatic progression and chromosomal instability in colon cancer. Genome research 2013, 23(9):1446-1461.

35. 35. Lu S, Dong W, Zhao P, Liu Z: IncRNA FAM83H-AS1 is associated with the prognosis of colorectal carcinoma and promotes cell proliferation by targeting the Notch signaling pathway. Oncology letters 2018, 15(2):1861-1868.

36. 36. Yang F, Lv S-X, Lv L, Liu Y-H, Dong S-Y, Yao Z-H, Dai X-X, Zhang X-H, Wang O-C: Identification of IncRNA FAM83H-AS1 as a novel prognostic marker in luminal subtype breast cancer. OncoTargets and therapy 2016, 9:7039.

37. 37. Zhang J, Feng S, Su W, Bai S, Xiao L, Wang L, Thomas DG, Lin J, Reddy RM, Carrott PW: Overexpression of FAM83H-AS1 indicates poor patient survival and knockdown impairs cell proliferation and invasion via MET/EGFR signaling in lung cancer. Scientific reports 2017, 7(1):1-10.

38. 38. Shan $H$, Yang $Y$, Zhu $X$, Han $X$, Zhang $P$, Zhang $X$ : FAM83H-AS1 is associated with clinical progression and modulates cell proliferation, migration, and invasion in bladder cancer. Journal of cellular biochemistry 2019, 120(3):4687-4693.

39. 39. Da J, Liu P, Wang R, Bu L: Upregulation of the long non-coding RNA FAM83H-AS1 in gastric cancer and its clinical significance. Pathology-Research and Practice 2019, 215(10):152616.

40. 40. Gong Y, Zou Y: Clinical significance of IncRNA FAM83H-AS1 in ovarian cancer. Eur Rev Med Pharmacol Sci 2019, 23:4656-4662.

41. 41. Michelotti EF, Michelotti GA, Aronsohn Al, Levens D: Heterogeneous nuclear ribonucleoprotein $\mathrm{K}$ is a transcription factor. Molecular and cellular biology 1996, 16(5):2350-2360. 
42. 42. Lee $\mathrm{M}-\mathrm{H}$, Mori $\mathrm{S}$, Raychaudhuri $\mathrm{P}$ : trans-Activation by the hnRNP K protein involves an increase in RNA synthesis from the reporter genes. Journal of Biological Chemistry 1996, 271(7):3420-3427.

43. 43. Yano $\mathrm{M}$, Okano $\mathrm{HJ}$, Okano $\mathrm{H}$ : Involvement of $\mathrm{Hu}$ and heterogeneous nuclear ribonucleoprotein $\mathrm{K}$ in neuronal differentiation through p21 mRNA post-transcriptional regulation. Journal of Biological Chemistry 2005, 280(13):12690-12699.

44. 44. Xiao Z, Ko HL, Goh EH, Wang B, Ren EC: hnRNP K suppresses apoptosis independent of p53 status by maintaining high levels of endogenous caspase inhibitors. Carcinogenesis 2013, 34(7):1458-1467.

45. 45. Yang J-H, Chiou Y-Y, Fu S-L, Shih I-Y, Weng T-H, Lin W-J, Lin C-H: Arginine methylation of hnRNPK negatively modulates apoptosis upon DNA damage through local regulation of phosphorylation. Nucleic acids research 2014, 42(15):9908-9924.

46. 46. Zhang K, Shi Z-M, Chang Y-N, Hu Z-M, Qi H-X, Hong W: The ways of action of long non-coding RNAs in cytoplasm and nucleus. Gene 2014, 547(1):1-9.

47. 47. Chu C, Zhang Qiangfeng C, da Rocha Simão T, Flynn Ryan A, Bharadwaj M, Calabrese JM, Magnuson T, Heard E, Chang Howard Y: Systematic Discovery of Xist RNA Binding Proteins. Cell 2015, 161(2):404-416.

48. 48. Zhang Z, Zhou C, Chang Y, Zhang Z, Hu Y, Zhang F, Lu Y, Zheng L, Zhang W, Li X: Long noncoding RNA CASC11 interacts with hnRNP-K and activates the WNT/ $\beta$-catenin pathway to promote growth and metastasis in colorectal cancer. Cancer letters 2016, 376(1):62-73.

49. 49. Li K, Sun D, Gou Q, Ke X, Gong Y, Zuo Y, Zhou J-K, Guo C, Xia Z, Liu L: Long non-coding RNA linc00460 promotes epithelial-mesenchymal transition and cell migration in lung cancer cells. Cancer letters 2018, 420:80-90.

\section{Figures}




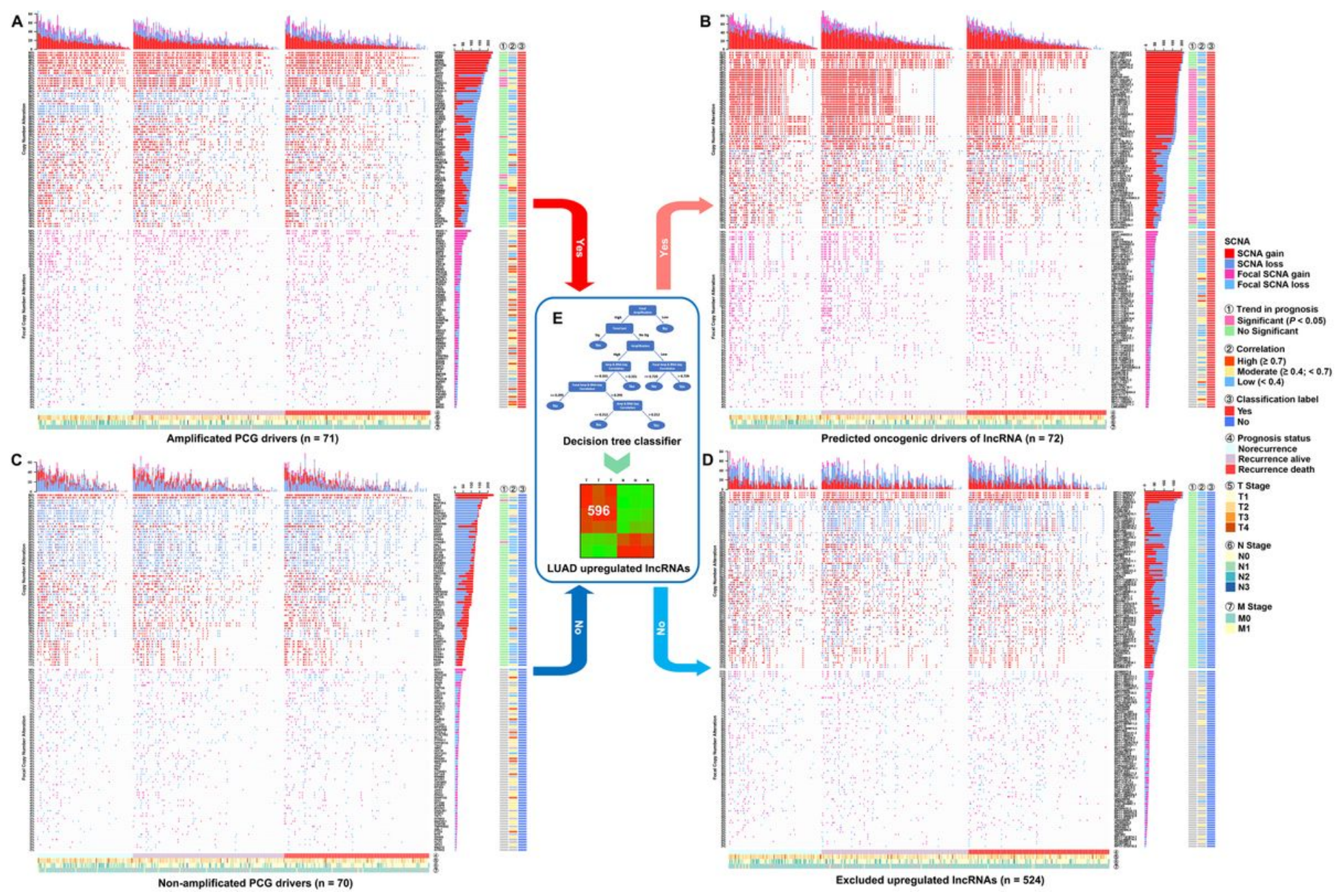

Figure 1

The input and output somatic copy number alteration profiles generated by the decision tree classifier. a.b Total SCNA and focal SCNA profiles of amplificated PCG drivers and predicted oncogenic drivers of IncRNAs. Red indicates an accumulation of SCNAs, while blue indicates a loss of SCNAs. Total SCNA gain or loss: The genomic segment value is greater than 0.2 or less than -0.2. Focal SCNA gain or loss: The GISTIC value is greater than 0.3 or less than -0.3 . Heatmaps were separated into three parts according to different prognosis statuses, and a trend test was conducted. Correlation estimation was based on Pearson's correlation coefficient between SCNA and expression levels; c.d Total SCNA and focal SCNA profiles of non-amplificated PCG drivers and excluded upregulated IncRNAs are shown (75 of 524 were randomly selected to present); e A constructed J48 decision tree was performed on LUADupregulated IncRNAs in LUAD. 
A

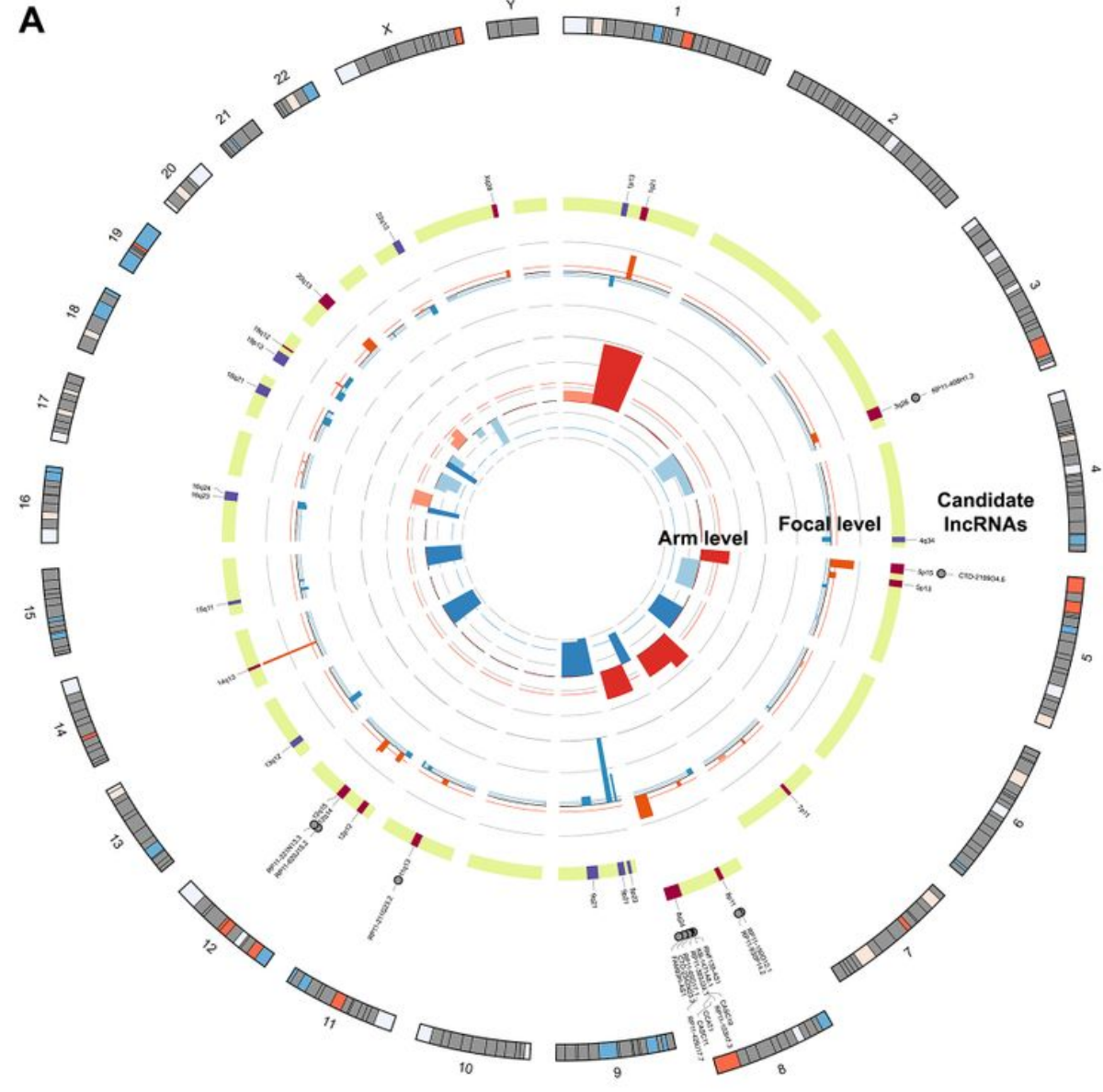

B

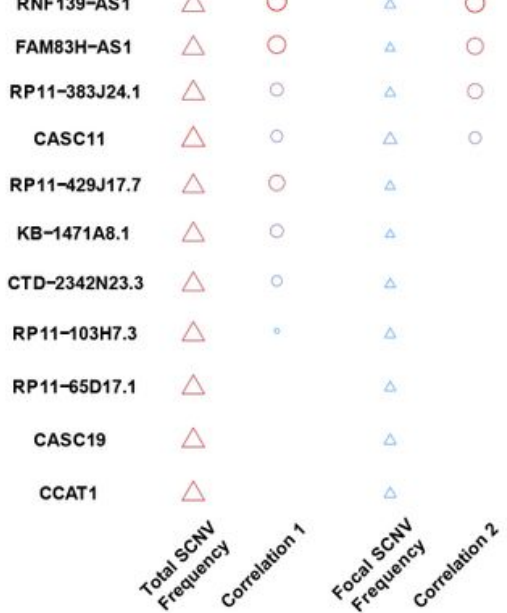

Correlation (rho)

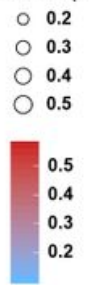

$\circ 0.3$

○ 0.4

0.5

0.5
0.4
0.3
0.2

Frequency

$\begin{aligned} \triangle & 0.2 \\ \triangle & 0.3\end{aligned}$

$\triangle 0.4$

$\triangle 0.4$
C
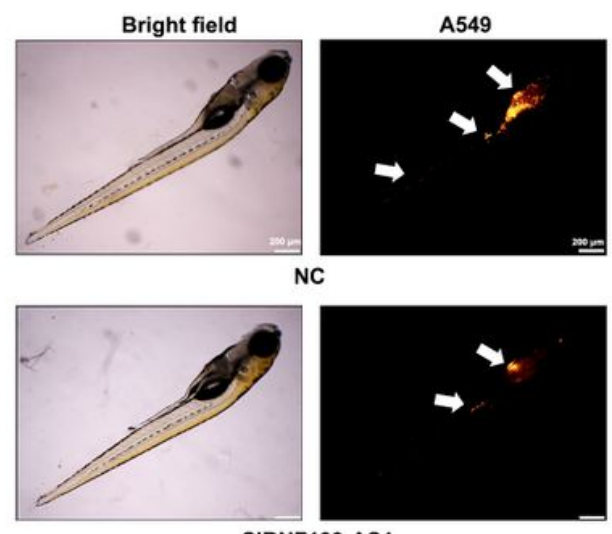

SiRNF139-AS1
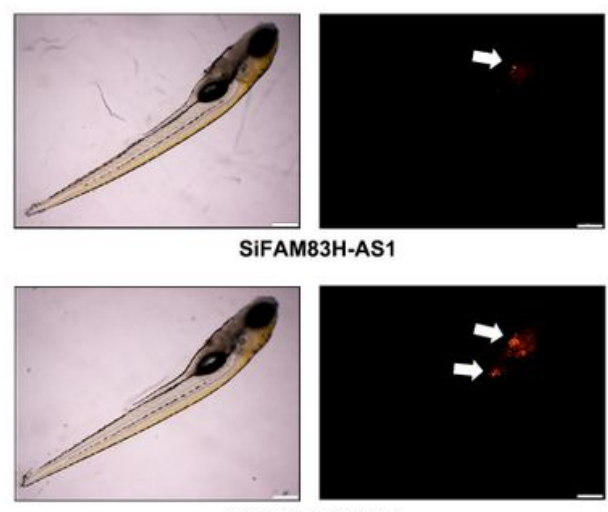

SiRP11-383J24.1

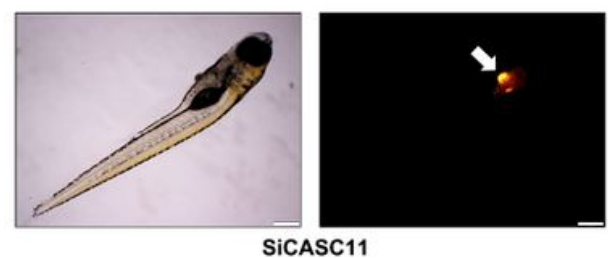

SiCASC11

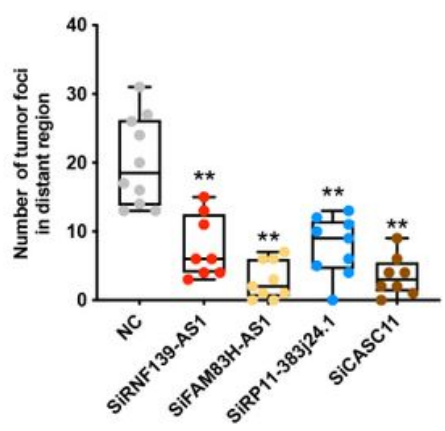

Figure 2

$8 q 24$ is a candidate oncogenic driver of IncRNA expression. a Predicted oncogenic drivers of IncRNAs located in high frequency amplified regions. The inner and middle circles represent arm- and focal-level SCNAs, respectively, and the outer circle indicates candidate IncRNAs in focal-level dominant regions; $b$ Detailed characteristics of 11 candidate IncRNAs located on chromosome 8q24 are shown. The SCNV frequency in LUAD and the corresponding correlation with expression were described; c Dil-red-labeled A549 cells in zebrafish embryos identified oncogenic functions that promoted proliferation and metastasis in RNF139-AS1, FAM83H-AS1, RP11-383j24.1 and RP11-429J17.7 in vivo (NC, $\mathrm{n}=10$ zebrafish/group; siRNF139-AS1, n = 8 zebrafish/group; siFAM83H-AS1, n = 9 zebrafish/group; siRP11- 
383j24.1, $n=9$ zebrafish/group; siCASC11, $n=8$ zebrafish/group). * $P<0.05$, and **, $P<0.01$. N. S, nonsignificant. Error bars, standard error of the mean.
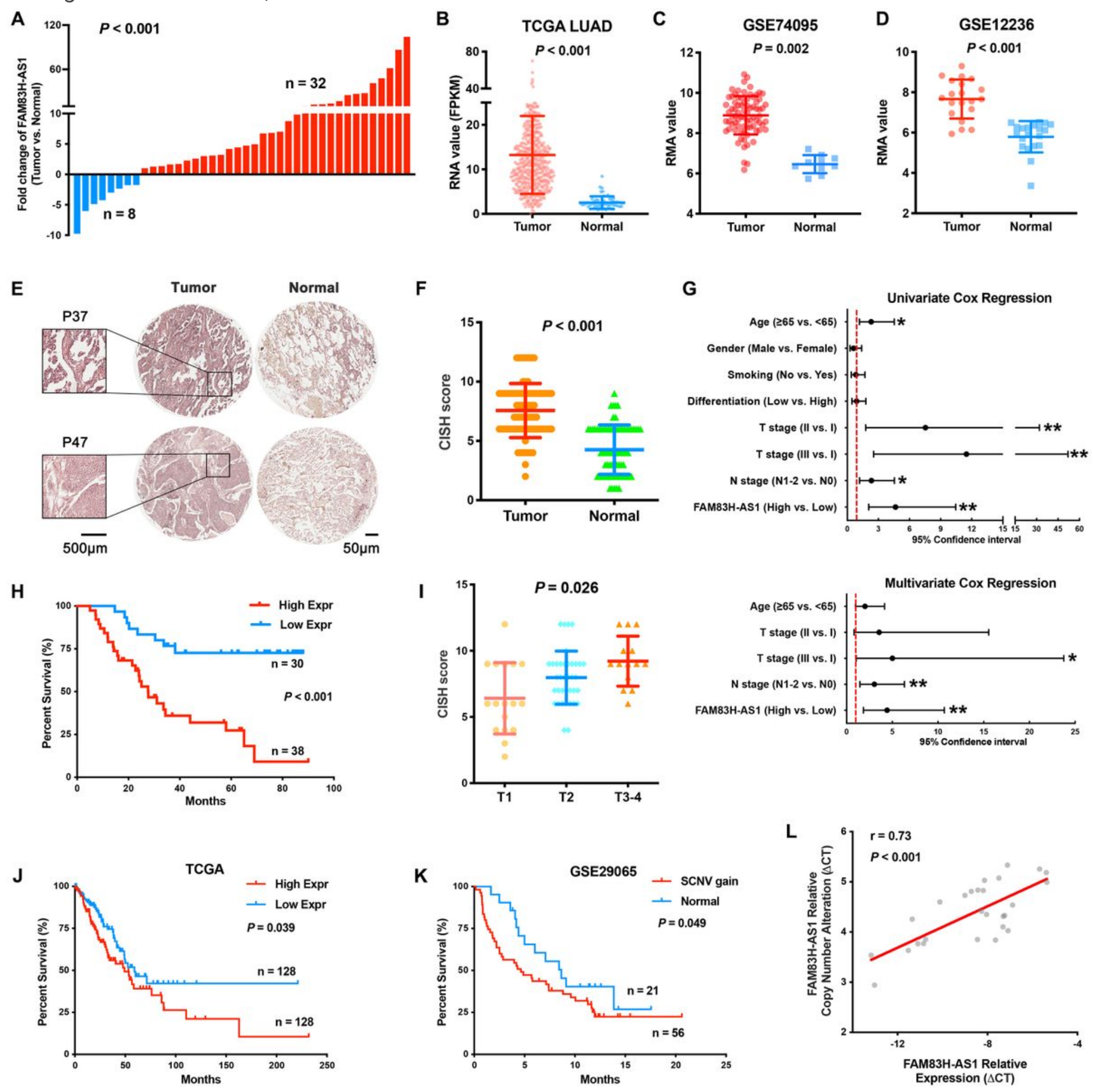

Figure 3

Clinical relevance of FAM83H-AS1 in lung adenocarcinoma. a qRT-PCR indicated that FAM83H-AS1 was upregulated in 32 pairs of 40 paired primary LUAD tissues in comparison to adjacent nontumor tissues; b.c.d Other independent cohorts all suggested overexpression of FAM83H-AS1 in LUAD tissues compared with adjacent nontumor tissues. FPKM, fragments per kilobase of exon model per million reads mapped; 
e.f The CISH results of the TMA of the JSCH cohort; $g$ Cox proportional hazards model indicated that the level of FAM83H-AS1 was an independent prognostic factor; h.i.j Higher expression of FAM83H-AS1 is associated with poor prognosis and greater tumor size both in JSCH and TCGA cohorts. The estimation cutoff value is based on median and quartile values in JSCH and TCGA cohorts separately; $\mathrm{k}$ SCNV gain of FAM83H-AS1 is related to poor survival in the TCGA cohort; I qRT-PCR results demonstrated a high correlation between gene expression and amplification of FAM83H-AS1 in LUAD tissues. *, P $<0.05$, and $\star \star, P<0.01$. Error bars, standard error of the mean.
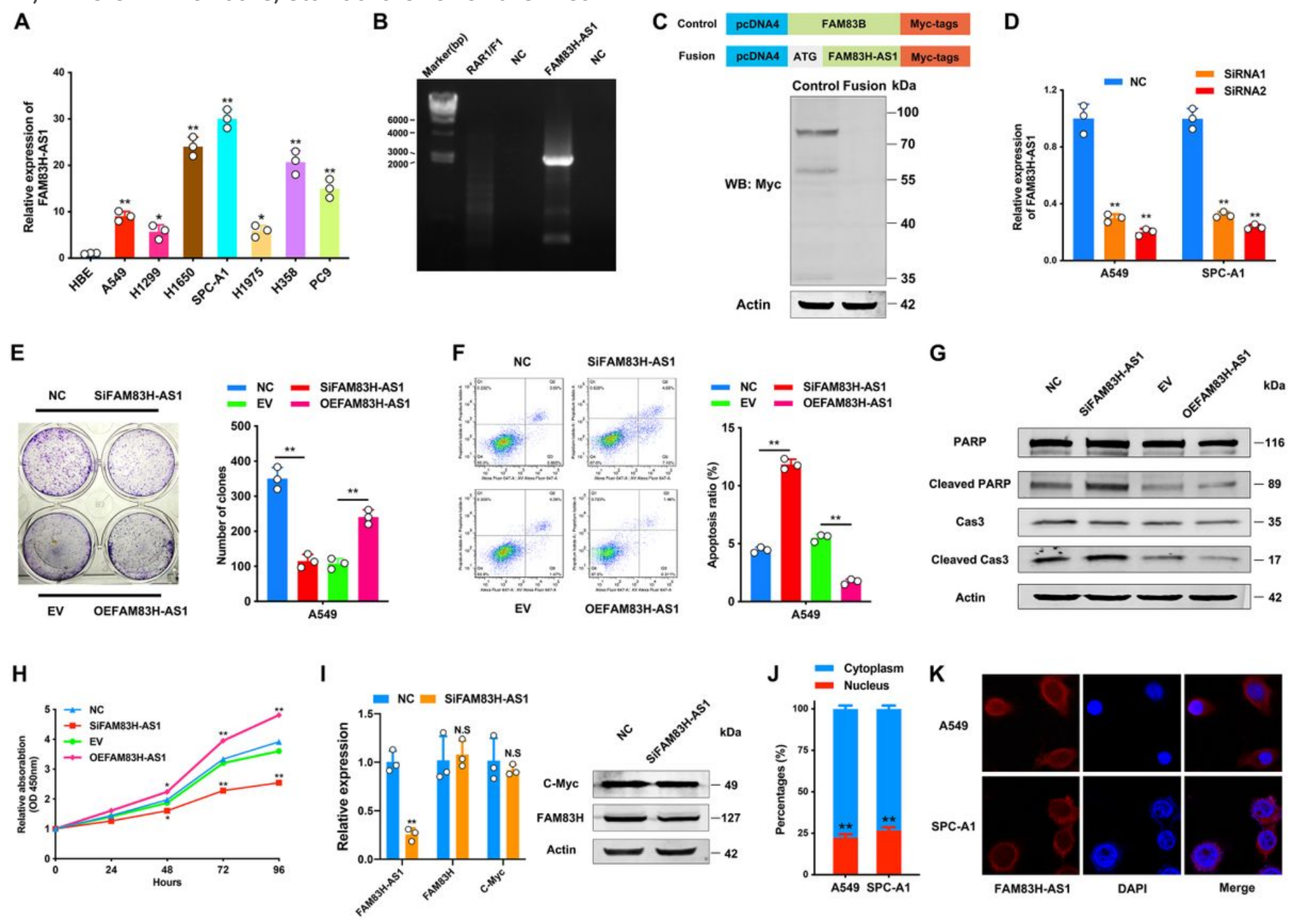

\section{Figure 4}

Gain and loss of function assays in vitro and features of FAM83H-AS1. a The expression of FAM83H-AS1 was detected in seven LUAD and normal HBE cell lines; $b$ The nested PCR product of RACE indicates an additional sequence of $295 \mathrm{nt}$ in the 5' end of reference NR_033849 and an additional $286 \mathrm{nt}$ in the 3' end; c Coding potentiality assay results indicated no translation of FAM83H-AS1. Full-length FAM83H-AS1 was cloned into pcDNA4/myc-his B with an N-terminal ATG codon. FAM83B served as a positive control. An anti-myc antibody was used to probe transcribed proteins; d SiRNA treatment reduced the expression level of FAM83H-AS1 in both A549 and SPC-A1 cells; e Colony formation assay of A549 cells; f.g Apoptosis assay by flow cytometry and analysis of PARP and Caspase-3 cleavage; h CCK-8 assay 
detected the proliferation of A549 cells; i qRT-PCR and western blotting demonstrated that FAM83H-AS1 did not regulate $\mathrm{C}-\mathrm{Myc}$ and $\mathrm{FAM} 83 \mathrm{H}$; j.k The nuclear mass separation and fluorescence in situ hybridization (FISH) assays suggested that FAM83H-AS1 was mainly distributed in the cytoplasm.

A
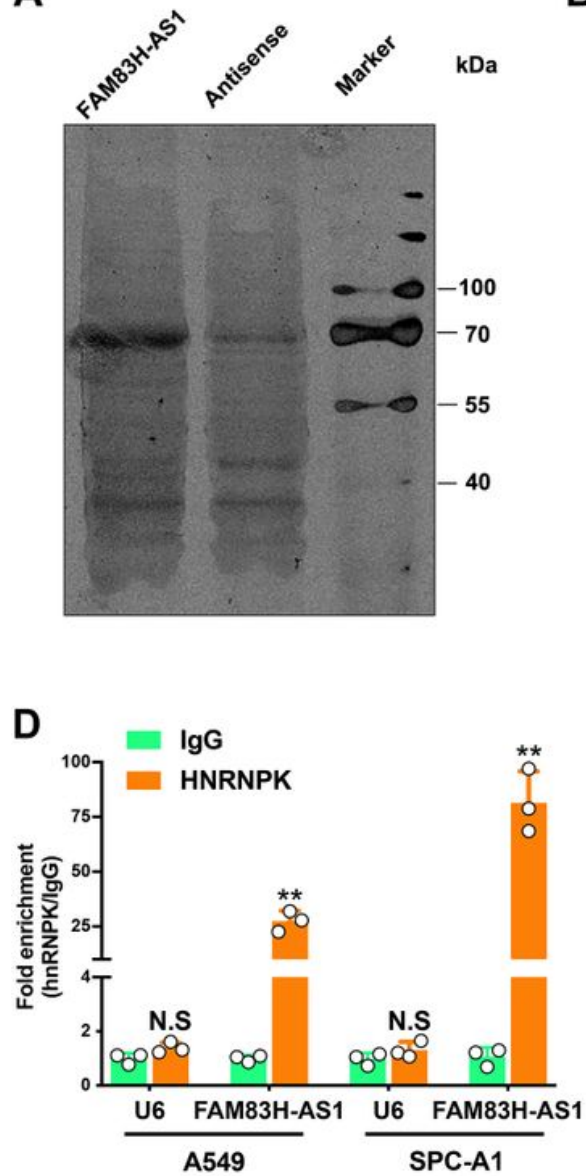

G

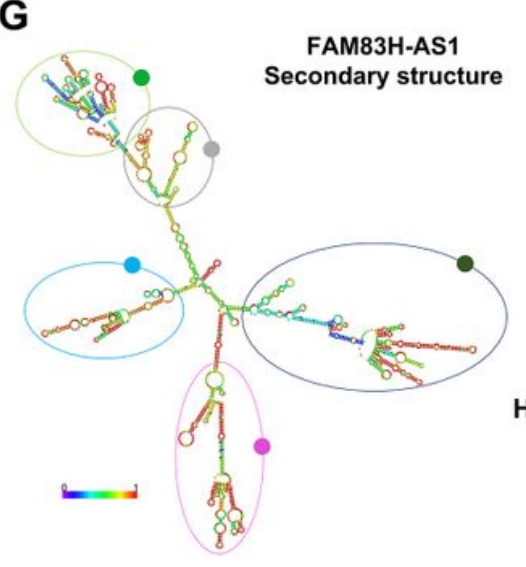

B

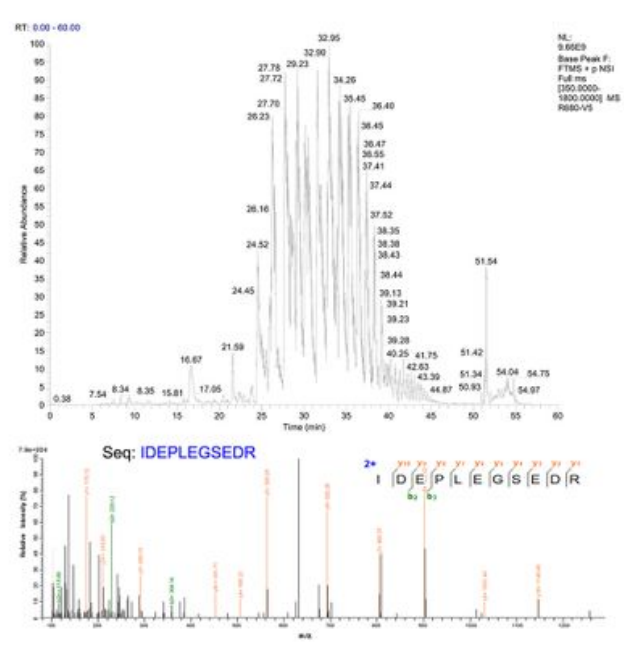

E
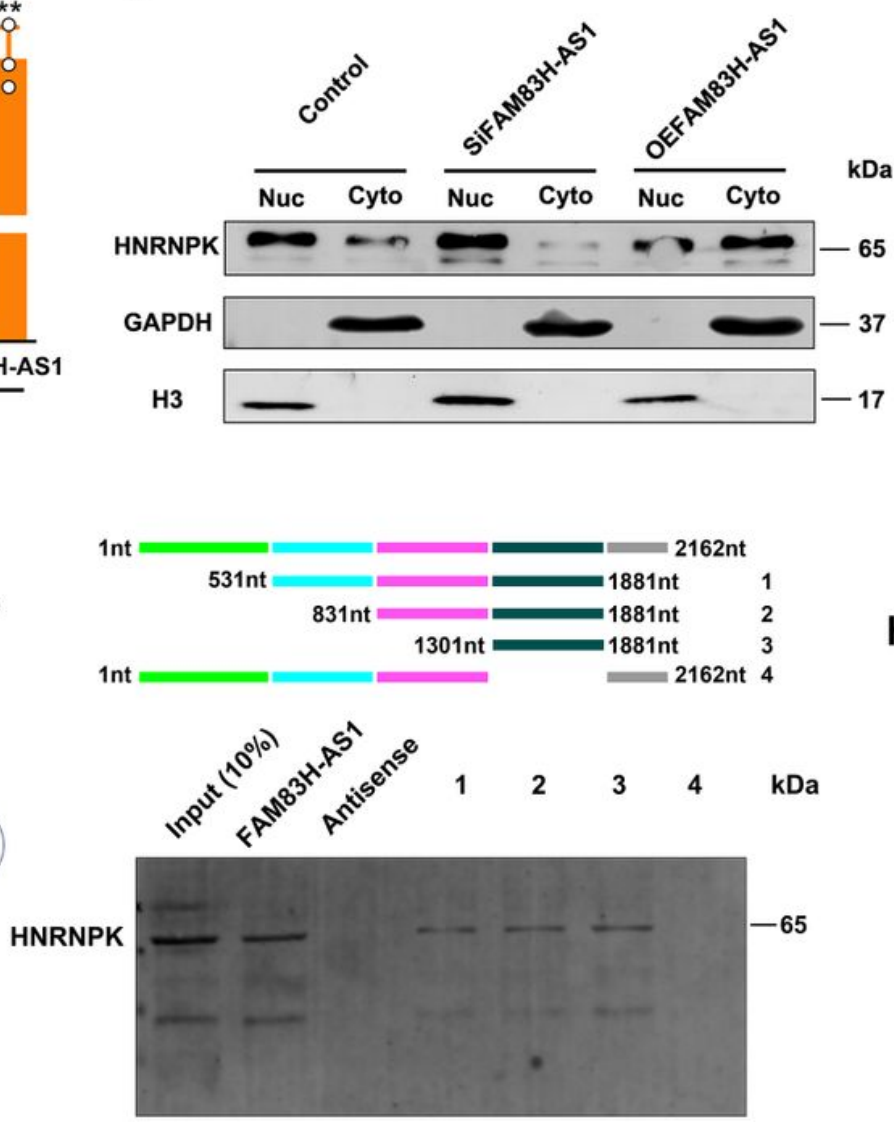

C

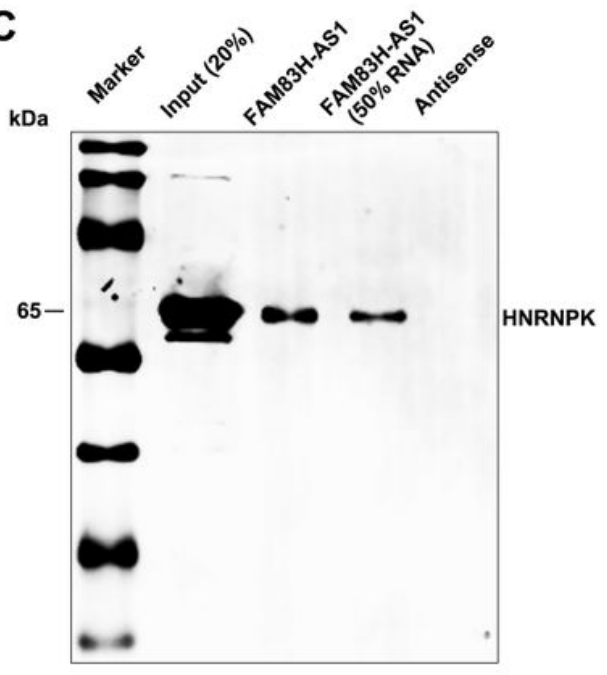

$\mathbf{F}$

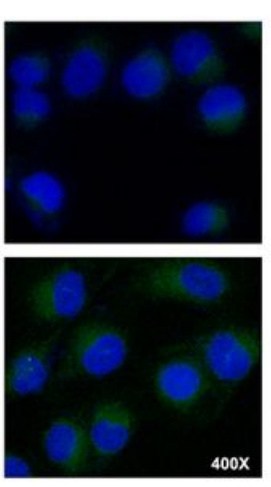

HNRNPK

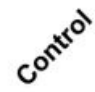

H

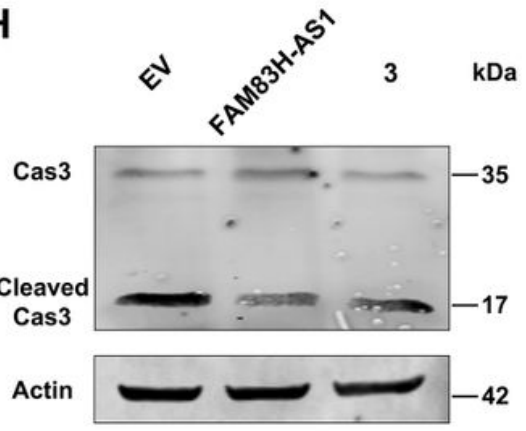

\section{Figure 5}

FAM83H-AS1 binds with HNRNPK to regulate lung adenocarcinoma cell apoptosis. a.b RNA pull-down and silver stain assays of biotinylated FAM83H-AS1-associated proteins. The FAM83H-AS1-specific band was excised and analyzed by mass spectrometry, which identified HNRNPK; c Western blot of proteins from pulldown assays; d RIP evaluation of the interaction between HNRNPK and FAM83H-AS1 using an 
anti-HNRNPK antibody ( $5 \mu \mathrm{g})$; IgG $(5 \mu \mathrm{g})$ served as a negative control. U6, U6 small nuclear RNA (snRNA); e Western blot of HNRNPK expression in the cytoplasm and nucleus of the indicated A549 cells. Scrambled sequences and empty vectors were combined as controls. Empty vector and scrambled sequences were added to the silencing and upregulation groups, respectively. GAPDH and Histone 3 served as loading controls; $f$ Immunofluorescence assays indicated an increase in cytoplasmic HNRNPK after increasing the expression of FAM83H-AS1 in A549 cells; $g$ The secondary structure of FAM83H-AS1 is shown as predicted by the centroid method (http://rna.tbi.univie.ac.at). The red color indicates strong confidence for the prediction of each base. RNA pull-down detection of the interaction between HNRNPK and FAM83H-AS1 truncations according to the predicted secondary structure; $\mathrm{h}$ Apoptosis assay of cells with the full-length and truncation mutant FAM83H-AS1, as assessed by Caspase-3 cleavage. *, $\mathrm{P}<0.05$, and $* \star, P<0.01$. N. S, nonsignificant. Error bars, standard error of the mean. 
A
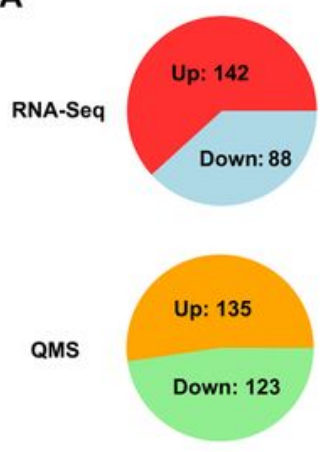

E

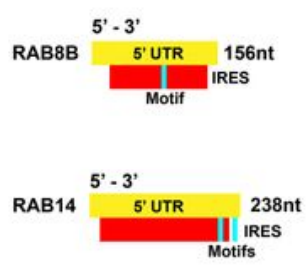

F
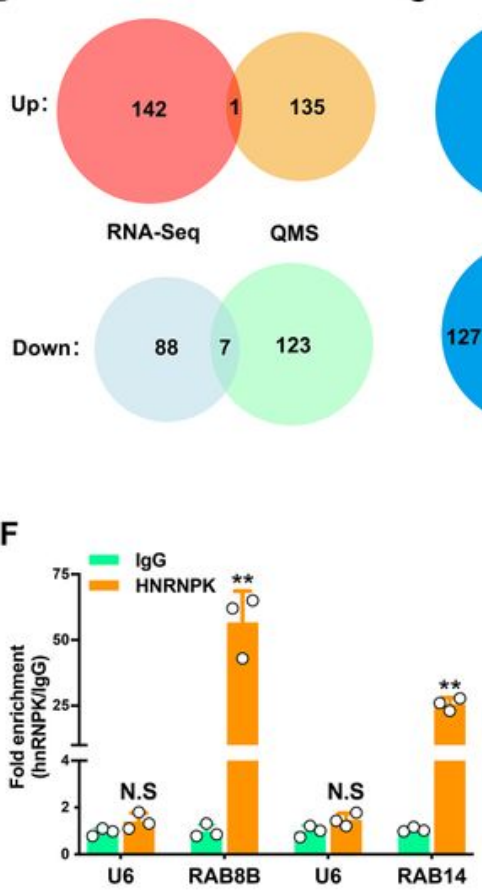

C
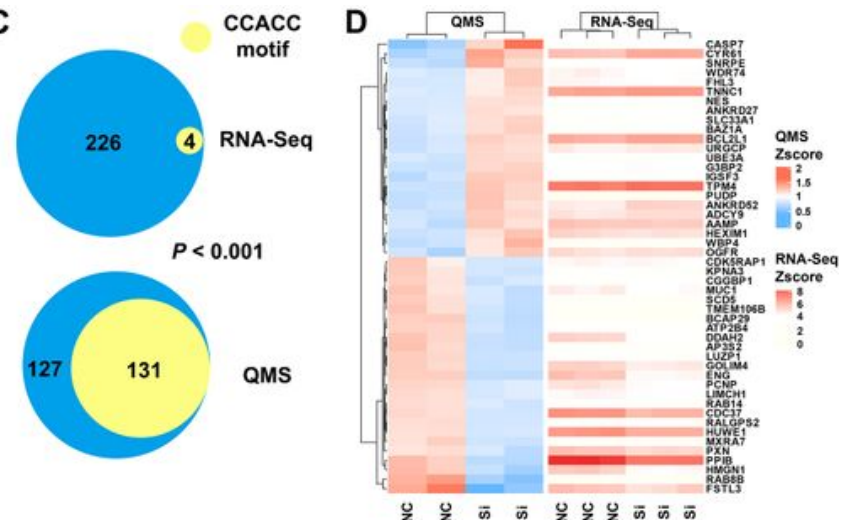

G

RAB8B - 5'UTR WT: CTCTCTCCACCGCCTCC MUT: СTCTCTGGTGGGCCTCC

RAB14 - 5'UTR
CAGCCACCAGC - . - GTGCCACC
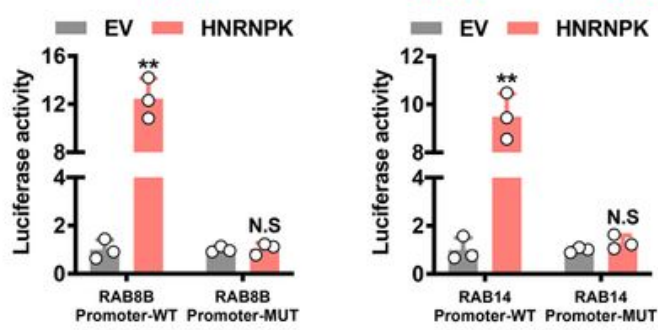

H
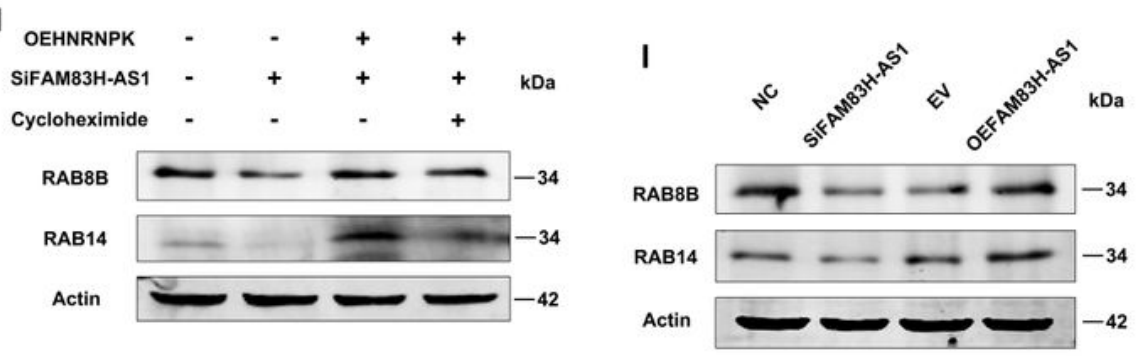

J

K
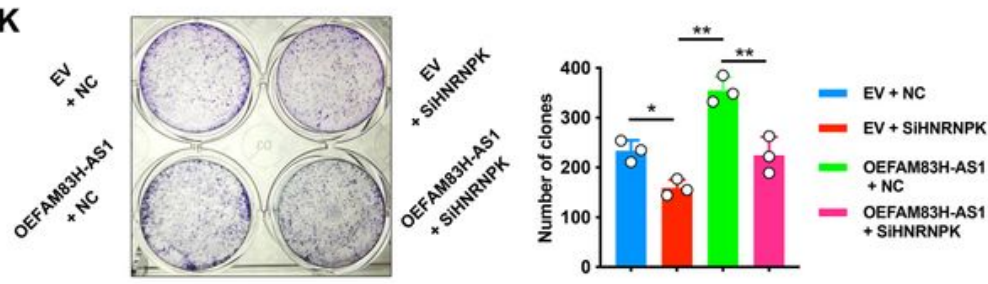

L
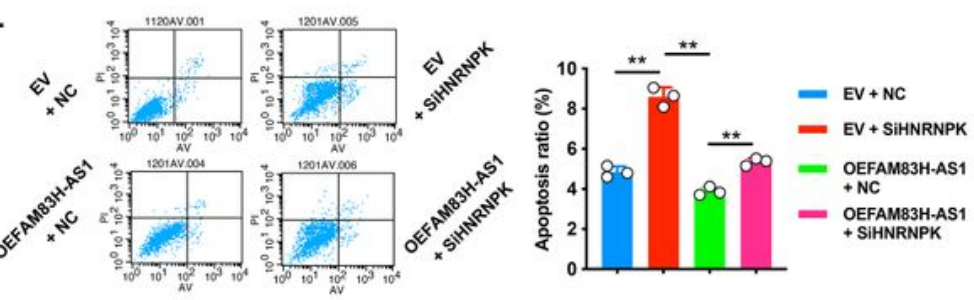

M
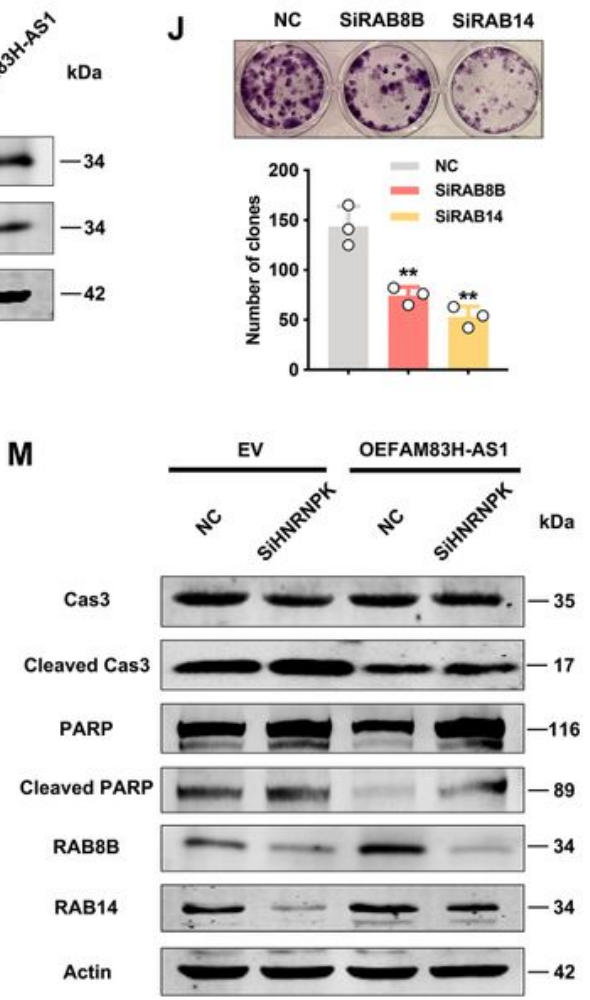

Figure 6

The FAM83H-AS1-HNRNPK complex coregulates the expression of RAB8B and RAB14. a Significantly differentially expressed genes identified by RNA-Seq (FDR $<0.01$ and fold change $>2$ ) and QMS (FDR < 0.05 and fold change > 1.2) after silencing FAM83H-AS1; $b$ Common upregulated and downregulated genes in the RNA-Seq and QMS results; c Significantly differentially expressed genes harboring HNRNPKspecific motifs in their 5'UTRs were identified by RNA-Seq and QMS results. P values were determined by 
Fisher's exact test; $d$ The top differentially expressed genes identified by QMS and the corresponding mRNA changes in RNA-Seq; e Predicted IRES sites (http://iresite.org) and identified HNRNPK motifs in the 5 'UTRs of RAB8B and RAB14; $f$ RIP evaluation of the interaction between HNRNPK and mRNAs of RAB8B and RAB14 using an anti-HNRNPK antibody as described above; $g$ Dual luciferase reporter assays showed that HNRNPK directly binds to the 5'UTRs of RAB8B and RAB14 and activates luciferase activity; $\mathrm{h}$ Effect of the translation inhibitor cycloheximide on the HNRNPK overexpression-induced increase in the protein levels of RAB8B and RAB14 in FAM83H-AS1 knockdown A549 cells; $i$ The silencing of FAM83HAS1 decreased the expression of RAB8B and RAB14, but overexpressing FAM83H-AS1 increased their expression; $j$ The colony formation assay results suggested an oncogenic function for RAB8B and RAB14 in A549 cells; $k$ Colony formation assays suggested that HNRNPK knockdown partially abolished the effects of FAM83H-AS1; I.m HNRNPK knockdown abolished the effects of FAM83H-AS1 on apoptosis, as revealed by flow cytometry and the cleavage of PARP and Caspase-3. The effect of HNRNPK knockdown on FAM83H-AS1 overexpression-induced protein expression of RAB8B and RAB14. *, $\mathrm{P}<0.05$, and $* \star, P<$ 0.01. N. S, nonsignificant. Error bars, standard error of the mean. 

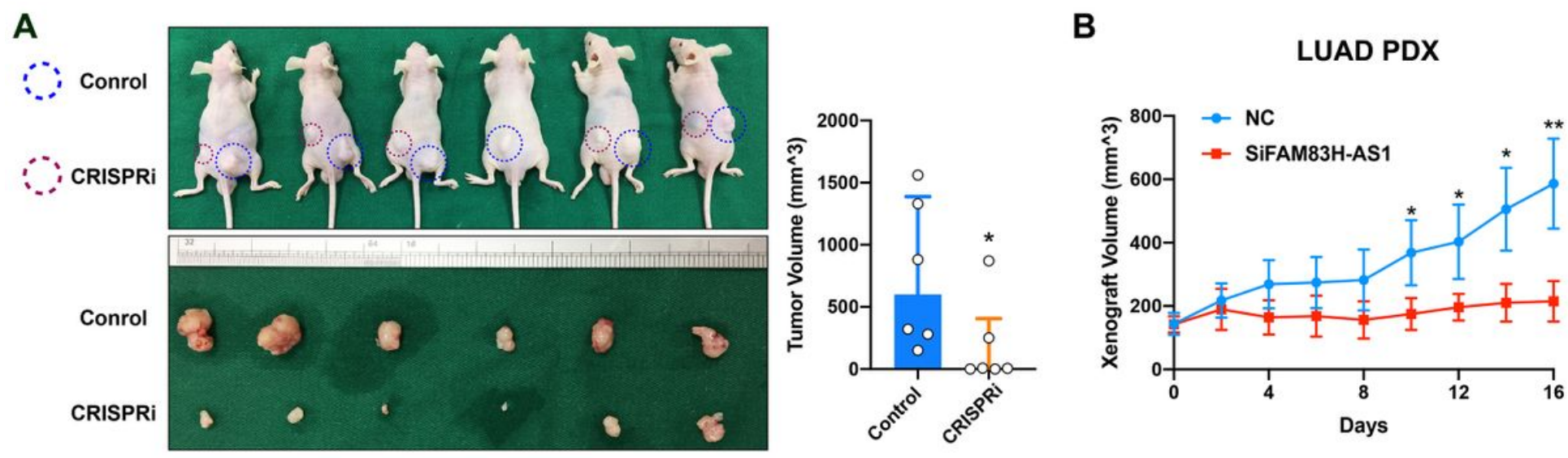

C

LUAD PDX

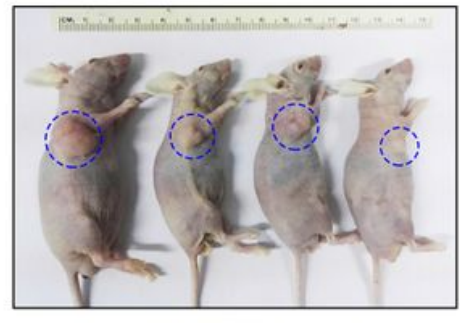

NC

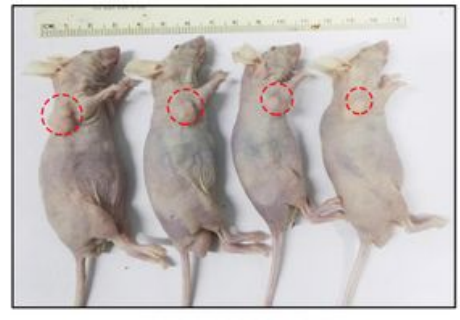

SiFAM83H-AS1

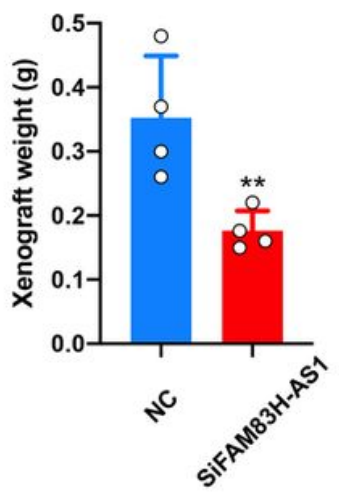

D
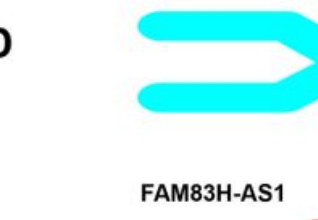

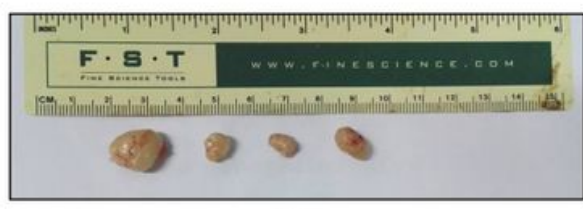

NC

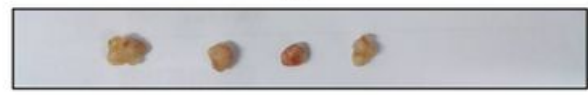

SiFAM83H-AS1

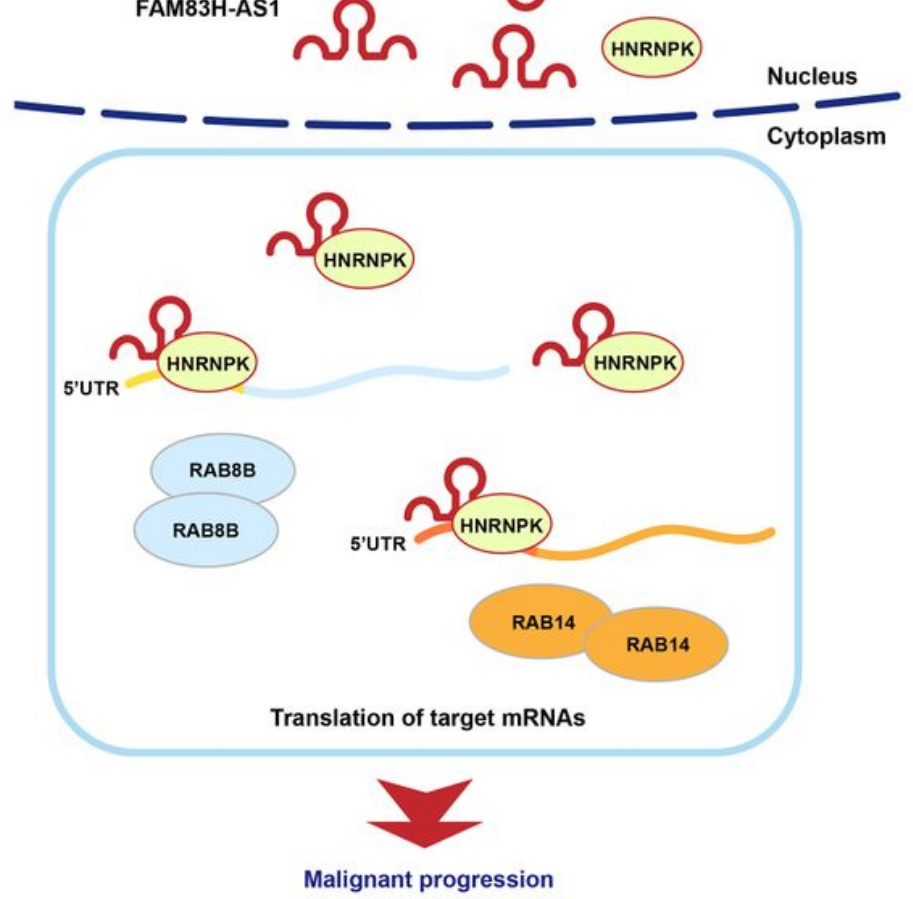

Figure 7

FAM83H-AS1 promotes lung adenocarcinoma tumorigenesis in vivo and acts as a promising therapeutic target. a Using CRISPRi technology to construct FAM83H-AS1 knockdown LUAD cells that were incorporated in xenograft tumor models showed that tumors grown from FAM83H-AS1 silenced cells were smaller than those grown from control cells; b.c PDTX model showing that intratumoral injection of siRNA targeting FAM83H-AS1 inhibited LUAD-derived tumor growth; d Schematic diagram of how FAM83H-AS1 promotes LUAD malignant progression. The chromosome 8q24 amplicon leads to overexpression of FAM83H-AS1 in LUAD, and the FAM83H-AS1-HNRNPK complex binds to the 5'UTRs of RAB8B and RAB14, activating target gene translation and suppressing apoptosis in LUAD cells.

\section{Supplementary Files}


This is a list of supplementary files associated with this preprint. Click to download.

- FigureS4.jpg

- FigureS4.jpg

- FigureS3.jpg

- FigureS3.jpg

- Figures2.jpg

- Figures2.jpg

- FigureS1.jpg

- FigureS1.jpg

- SuppleTables.xlsx

- SuppleTables.xlsx 